\title{
Impact of rotating and fixed nozzles on vortex breakdown in compressible swirling jet flows
}

\author{
T. Luginsland ${ }^{\mathrm{a}, *}, \mathrm{~F}_{\text {. Gallaire }}{ }^{\mathrm{b}}$, L. Kleiser $^{\mathrm{a}}$ \\ ${ }^{a}$ Institute of Fluid Dynamics, ETH Zurich, 8092 Zurich, Switzerland \\ ${ }^{\mathrm{b}}$ Laboratory of Fluid Mechanics and Instabilities, EPF Lausanne, 1015 Lausanne, Switzerland
}

\section{A R T I C L E I N F O}

\section{Article history:}

Received 17 November 2014

Received in revised form

22 October 2015

Accepted 12 January 2016

Available online 29 January 2016

\section{Keywords:}

Swirling flows

Vortex breakdown

Helical instabilities

Nozzle modelling

\begin{abstract}
A B S T R A C T
Vortex breakdown of swirling, round jet flows is investigated in the compressible, subsonic regime by means of Direct Numerical Simulation (DNS). This is achieved by solving the compressible Navier-Stokes equations on a cylindrical grid using high-order spatial and temporal discretization schemes. The Reynolds number is $\operatorname{Re}=\rho_{c}^{\circ} w_{c}^{\circ} R^{\circ} / \mu_{c}^{\circ}=5000$ and the flow is moderately compressible with Mach number $M a=w_{c}^{\circ} / \sqrt{\gamma R_{\mathrm{air}}^{\circ} T_{c}^{\circ}}=0.6$. The integral swirl number at the inflow is $S_{\text {int }}=0.85$. The parameters are chosen properly so as to make comparisons with existing experiments at lower Mach numbers possible while still enabling a study of compressible and baroclinic effects. Different from previous numerical investigations, a nozzle immersed in the fluid is included in the computational domain and is modelled as an isothermal no-slip wall, either rotating with the mean azimuthal flow direction or kept at rest. The present investigation aims to clarify the role played by the nozzle wall motion for the vortex breakdown of the swirling jet. We study the nozzle flow as well as the swirling jet flow simultaneously, a novelty for numerical investigations of vortex breakdown in swirling jets. Depending on the nozzle wall motion, the flow differs significantly upstream of the vortex breakdown: for the rotating nozzle, the flow inside the nozzle is purely laminar and the azimuthal boundary layer at the outer nozzle wall gives rise to the axisymmetric mode $n=0$ and a single-helix type instability with azimuthal wave number $n=1$. With the nozzle at rest, a transitional flow is observed within the nozzle where a helical instability with azimuthal wave number $n=12$ dominates, growing in the boundary layer at the nozzle wall. For both nozzle setups, the helical instabilities observed for the nozzle flow interact with the developing vortex breakdown and the conical shear-layer downstream of the nozzle. For the nozzle at rest, this interaction results in a vortex breakdown configuration which is shifted in the upstream direction and which has a smaller radial and streamwise extent compared to the rotating nozzle case and the recirculation intensity is higher. The dominant frequency is highly influenced by the flow upstream of the vortex breakdown and is substantially higher for the nozzle at rest. Although the nozzle flow field differs for the two configurations and therefore alters the vortex breakdown downstream, a single-helix type instability $n=1$ governing the vortex breakdown is found for both cases. This provides strong evidence for the robustness of the instability mechanisms leading to vortex breakdown.
\end{abstract}

(c) 2016 Elsevier Masson SAS. All rights reserved.

\section{Introduction}

The physical phenomenon of vortex breakdown occurs in many technical applications, e.g. on delta wing aircraft [1] and in vortex burners [2], and can also be observed in nature [3]. A field of ongoing research is swirling jet flows undergoing vortex breakdown [4]. For a sufficiently high circumferential velocity

\footnotetext{
* Corresponding author.

E-mail address: luginsland@ifd.mavt.ethz.ch (T. Luginsland).
}

relative to the streamwise velocity vortex breakdown occurs. The flow state of vortex breakdown is characterized by a finite region of strong recirculation near the centreline of the swirling flow and a high radial spreading rate [5]. Helical instabilities of coand counter-rotating type, winding against or in the mean flow direction, dominate the flow field [6]. It is of great interest to understand the fundamental features of vortex breakdown, to know the parameters at which vortex breakdown occurs, and to get insight into possible control mechanisms for this special flow configuration. Although in more than five decades of intense research many attempts were made to explain vortex breakdown, 
a commonly accepted theory of the underlying mechanisms is still missing. For reviews of the vortex breakdown phenomenon, we refer to Lucca-Negro and O'Doherty [7] and references therein.

Experimental investigations reported in the literature vary in the design of the devices used for generating the swirling jet flow as well as whether the nozzle wall itself is kept at rest or rotating. The studies mainly split up in two groups, either utilizing a long rotating pipe with a straight or contracting nozzle attached, e.g. Liang and Maxworthy [8] and Facciolo et al. [9], or a pipe and a nozzle at rest in combination with a rotating honeycomb or guiding vanes to generate swirl, e.g. Billant et al. [5], Gallaire et al. [10], Oberleithner et al. [4] and Leclaire and Jacquin [11].

Liang and Maxworthy [8] and Liang and Maxworthy [12] used a long rotating pipe attached to a large water tank to generate swirling jets. The authors conducted experiments at Reynolds numbers up to $R e_{D}=2000$ (based on the bulk velocity). The streamwise velocity profile was initially uniform and laminar. Helical modes $n=+2,+3$ (co-rotating, counterwinding) were found to be dominant before vortex breakdown, while after vortex breakdown modes $n=+1,+2$ competed with each other, with mode $n=+1$ being most unstable. In the post-breakdown stage the dominant modes were suggested to be self-excited/globally unstable, a behaviour identified as a super-critical Hopf-bifurcation: the saturation amplitude of modes $n=+1,+2$ depended linearly on the critical swirl parameter, cf. Huerre and Monkewitz [13]. The flow criticality and shearlayer morphology remained unchanged with Reynolds number. The authors concluded that the swirl difference of the jet to the ambient fluid had only a minor effect on the flow criticality, which depended mainly on the velocity distribution of the vortex core. The effects of the developing boundary layer at the outer nozzle wall on the vortex breakdown of the swirling jet were not discussed.

Oberleithner et al. [4] conducted experiments in air at a Reynolds number $R e_{D}=20,000$ (based on the bulk velocity) of a turbulent, swirling jet over a range of swirl numbers. The authors focused on the description of three-dimensional coherent structures by means of POD and compared the empirical results to results from a weakly non-parallel spatial linear stability analysis. In this stability analysis the Reynolds number, $R e$, and the complex axial wave number, $\alpha$, were assumed to vary in the streamwise direction. The analysis was carried out on subsequent downstream positions to account for the non-parallel base flow assuming the instabilities in the outer shear-layer to be of convective type. A frequency was measured which dominates the entire flow field. The global dominant frequency was used as input parameter for the stability investigation. As in the earlier work by Liang and Maxworthy [8], evidence was found for the existence of a super-critical Hopf bifurcation on which a global mode could get established. The global mode was identified to be a corotating, counter-winding single-helix which was triggered by the precessing of the vortex core in the inner region of the jet.

Due to the differences in the experimental setups, the flow upstream of the nozzle end section differs as well. For the first group of experiments, the azimuthal velocity component at the wall is identical to the rotation speed of the nozzle, while for the latter the fluid is at rest at the nozzle wall. Differences in the experimental setups make it difficult to compare results of various studies, especially because the flow state upstream of vortex breakdown within the nozzle is rarely documented. Some discussion is found in Facciolo et al. [9] and Leclaire and Jacquin [11], but is restricted to flows before the onset of vortex breakdown.

Facciolo et al. [9] investigated turbulent swirling pipe flows as well as jet flows, both experimentally and numerically, and focused on the turbulent properties of the flows. Both flow regions - the pipe and the jet flows - were studied separately and an interaction of the pipe flow and the swirling jet flow was neglected in the numerical investigations and not discussed for the experimental results. Since the swirl intensity was below the threshold for vortex breakdown, no upstream effects of the swirling jet on the pipe flow were considered. The authors found that the fully developed flow within the rotating pipe was not in solid-body rotation due to the Reynolds shear stresses, which is in agreement with findings reported in Orlandi and Fatica [14]. A counter-rotating core was observed in the swirling jet flow six-to-eight pipe diameters downstream of its end section. The azimuthal velocity profile of the mean flow changed sign in the vicinity of the jet centreline developing a counter-rotation for $r \leq 0.5$.

Leclaire and Jacquin [11] reported on high-Reynolds number rotating flows in a pipe with a final contraction. The authors found that vortex breakdown within the pipe is suppressed by the contraction at the pipe end and observed instead standing axisymmetric Kelvin waves (see, for instance, Saffman [15]) to be present. The observations made were independent of the employed contraction ratio. The authors observed a high fluctuation level in the pipe exit plane and a spiralling motion, both connected to the flow upstream of the pipe exit plane and its change in criticality from super-critical to sub-critical (see Benjamin [16] and Lucca-Negro and O'Doherty [7] for a review). To avoid this change in criticality already within the pipe and to guarantee smooth flow conditions in the pipe exit, especially at high swirl, Leclaire and Jacquin [11] suggested to exclude a final contraction in swirling jet flow experiments. Although the swirling jet flow was not studied in their investigation, it gave strong evidence of the importance of the upstream flow conditions within the pipe on the vortex breakdown configuration.

Numerical investigations of vortex breakdown in swirling flows in the compressible regime have been published by Melville [17], Müller and Kleiser [18] and Luginsland and Kleiser [19]. Melville [17] solved the compressible Euler equations to study vortex breakdown of a subsonic free vortex of Burgers' type. The suppressing effect of an increased Mach number on the vortex breakdown reported in [20] was reversed leading to a promotion and an upstream shift of the recirculation region. The vortex breakdown configuration was governed by a double-helix type structure, co-rotating with the mean flow and winding in the opposite direction, but different from the structure observed by Sarpkaya [21]. In the study by Müller and Kleiser [18], natural and forced swirling jets at $R e=5000, M a=0.6$ were considered and linear stability analysis was applied to identify unstable modes. The results showed good qualitative agreement with the experimental observations reported in [8]. At sufficiently high swirl the jet broke down and a conical breakdown state established with a pronounced recirculation zone around the jet axis. Counter-rotating, co-winding single- and double-helix type instabilities dominated the flow field. The more rapid breakdown of the jet and the stronger deceleration of the streamwise velocity at the jet centreline compared to results reported in [8] was suggested to be linked to compressibility effects. The swirling jet flow regime was considered only and a nozzle was not included into the computational domain.

In their recent numerical study Luginsland and Kleiser [19] investigated compressibility effects on swirling jet flows undergoing vortex breakdown. The swirling jets were emanating from a rotating nozzle. Compressibility effects were studied for Mach numbers in the range $0.4 \leq M a \leq 0.8$ at a constant inflow temperature and inflow swirl rate in the nozzle end section (for additional information see also [22]). The authors found a promotion of vortex breakdown for increasing Mach numbers and confirmed the results reported in [17]. The delaying effect of compressibility 
on the onset of vortex breakdown reported in [23] was counteracted by jet-to-ambient density/temperature ratio effects. Nevertheless, the growth of helical instabilities was damped for increasing Mach numbers, an observation in accordance with findings reported in [24].

In the present investigation, we study swirling nozzle-jet flows to bridge the gap in the investigations reported in the literature, where swirling pipe and jet flows are studied separately without allowing for an interaction of the two. The nozzle flow and the swirling jet flow are therefore investigated simultaneously in the present contribution. In addition, we investigate swirling nozzle-jet flows for the two cases of a rotating nozzle and a nozzle kept at rest. This allows to provide a link between the two groups of experimental investigations found in the literature, where either a rotating nozzle or a nozzle at rest is considered.

We use a numerical framework $[22,25,19,26,24]$, which allows for a precise study of the effects of the nozzle configuration on the flow field due to high-order methods in time and space. Our aim is to provide insight into the instability mechanisms inherently connected to the nozzle either rotating with the mean flow or kept at rest. Furthermore, we report on the effects of the flow within the nozzle on the vortex breakdown behaviour of the swirling jet for the two nozzle configurations.

This paper is organized as follows. In Section 2 we briefly introduce the numerical framework and computational aspects of the present study. Setup differences for the rotating nozzle and the nozzle at rest are discussed in Section 3. In Section 4 numerical results are presented and compared to results reported in the literature. We summarize our study, discuss the main findings and conclude the investigation in Section 5.

\section{Numerical framework}

In this section, we summarize the basic approach and the numerical methods used in the present investigation. An extensive documentation is given in Müller [24]. The radial, azimuthal and streamwise coordinates and velocities are denoted by $r, \theta, z$ and $u, v, w$, respectively. The compressible, three-dimensional Navier-Stokes equations are solved numerically on a cylindrical domain of dimension $L_{r} \times L_{\theta} \times L_{z}=10 R^{\circ} \times 2 \pi \times 20 R^{\circ}$, see Fig. 1 for a schematic sketch of the setup ( ${ }^{\circ}$ denotes dimensional quantities). The governing equations are non-dimensionalized by the nozzle inner radius $R^{\circ}$ and centreline quantities at inflow, which are denoted by the subscript ()$_{c}$. The Reynolds number is defined as $R e=\rho_{c}^{\circ} w_{c}^{\circ} R^{\circ} / \mu_{c}^{\circ}=5000$, where $w^{\circ}$ is the streamwise velocity, $\rho^{\circ}$ is the density and $\mu^{\circ}$ is the dynamic viscosity. The Reynolds number is kept constant throughout the present investigation because no significant changes in the flow field, especially in the velocity fluctuation levels inside the boundary and shear-layers, were observed in the range $(2500 \leq R e \leq 7500)$ for the setup with a rotating nozzle. This result is in agreement with the observation that the mechanisms leading to vortex breakdown are mainly of non-viscous nature [27] and independent of the Reynolds number over a wide parameter range $[28,29]$. For much higher Reynolds numbers ( $R e \geq 100,000)$ though, strong effects on the vortex breakdown may be expected [30]. The present choice of the Reynolds number $R e=5000$ leads to a level of velocity fluctuations which is within $\pm 5 \%$ of the fluctuation levels observed in recent studies reported in literature $[8,9,31]$ as well as to similar radial distributions. The Reynolds numbers of these investigations differ significantly, giving further evidence for the minor effects of the Reynolds number on vortex breakdown and thus for the generality of the present results. The Mach number is chosen as $\mathrm{Ma}=w_{c}^{\circ} / \sqrt{\gamma R_{\mathrm{air}}^{\circ} T_{c}^{\circ}}=0.6$ where $\gamma=1.40$ is the ratio of specific heats, $R_{\text {air }}^{\circ}=287 \mathrm{~J} / \mathrm{kg} / \mathrm{K}$ is the gas constant of air and $T^{\circ}$ is the temperature. (At sea-level conditions, the nozzle radius

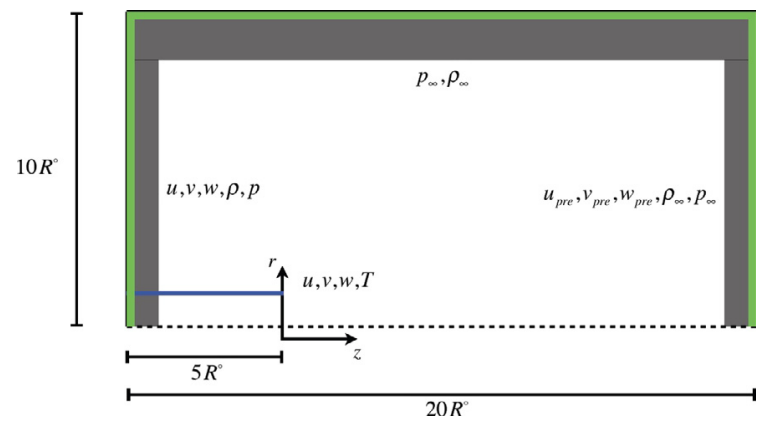

Fig. 1. Computational domain for simulations. Non-reflecting boundary conditions marked in green. Sponge layers are shaded in grey. The nozzle wall is indicated in blue. (For interpretation of the references to colour in this figure legend, the reader is referred to the web version of this article.)

corresponds to approximately $R^{\circ}=0.4 \mathrm{~mm}$ and the centreline velocity to approximately $w_{c}^{\circ}=200 \mathrm{~m} / \mathrm{s}$.) Following Müller and Kleiser [18] the Mach number is chosen in the moderately compressible regime, so as to make comparisons with existing experiments at lower Mach numbers possible while still enabling a study of compressible and baroclinic effects.

Our numerical code PARACONCYL $[22,25]$ utilizes high-order spatial finite-difference schemes with up to 10th order accuracy [32] in the streamwise and the radial directions. In the circumferential direction, a Fourier pseudo-spectral method is used to calculate the spatial derivatives. A fourth-order Runge-Kutta scheme [33] is employed for time integration. The numerical code has been validated extensively in previous investigations [18,34, $25,22,24]$. A nozzle is included in the computational domain which is modelled as an isothermal, no-slip wall to study the effects of the upstream flow on vortex breakdown and to allow for an interaction of the nozzle and the jet flow. The nozzle length is $L=5$ and the nozzle wall thickness is $d=\left(R_{o}^{\circ}-R^{\circ}\right) / R^{\circ}=0.1$ where $R_{o}^{\circ}$ denotes the outer nozzle radius. The nozzle wall is straight in accordance with suggestions made by Leclaire and Jacquin [11].

At the computational domain boundaries, non-reflecting conditions [35] are implemented, cf. Fig. 1. The non-reflecting conditions are supplemented with sponge layers [36] for five variables $(\rho, p, u, v, w)$ at the inflow and outflow and for pressure and density $(p, \rho)$ in the far-field, respectively. The variables $(\rho, p, u, v, w)$ are thereby non-dimensionalized w. r. t. centreline quantities. The imposed reference solution at the outflow is obtained from a precursor simulation. At the nozzle wall three velocities and the temperature are prescribed. The setup of boundary conditions as well as the initialization follows the recommendations given in [26] and is discussed in more detail in [22].

To obtain the results presented in Section 4 the simulations were run with a resolution of $N_{r} \times N_{\theta} \times N_{z}=480 \times 288 \times 480$ grid points. A grid resolution study is documented in Luginsland and Kleiser [26], providing evidence of the convergence of mean flow results. The time-step is chosen as $\Delta t=0.002$ according to a Courant-Friedrichs-Lewy criterion [24]. The simulations were performed as Direct Numerical Simulations (DNS) with mild filtering of the flow variables applied every second time-step to stabilize the simulation, cf. Müller [24]. The simulations were run in parallel on 768 cores on a CRAY XE6 supercomputer (Monte Rosa) at the Swiss National Supercomputing Centre (CSCS), Lugano.

\section{Setup differences for the rotating nozzle and the nozzle at rest}

We perform numerical simulations for compressible swirling jet flows undergoing vortex breakdown with either a rotating nozzle or a nozzle at rest included in the computational domain. 
The rotating nozzle setup follows Liang and Maxworthy [8] and Facciolo et al. [9], who utilized a rotating pipe to generate swirl. The nozzle at rest follows Billant et al. [5] and Oberleithner et al. [4], who used either guiding vanes or a rotating honeycomb for swirl generation. For both configurations, we define inflow boundary conditions for the pressure, $p$, and the density, $\rho$, by integrating the radial momentum equation and using the equation of state for an ideal gas, $\gamma M a^{2} p=\rho T$, assuming a uniform temperature distribution $T(r)=1[18,26]$ :

$$
\begin{aligned}
& p(r)=\frac{1}{\gamma \cdot M a^{2}} \cdot \exp \left(\int_{r^{\prime}=0}^{r} \frac{\gamma \cdot M a^{2} \cdot v^{2}\left(r^{\prime}\right)}{T \cdot r^{\prime}} d r^{\prime}\right), \\
& \rho(r)=\frac{p(r) \cdot \gamma \cdot M a^{2}}{T} .
\end{aligned}
$$

This choice leads to an initial radial stratification of the density and the pressure which may lead to the presence of baroclinic effects, as discussed in Section 4. The radial and streamwise velocity components at the inflow are defined as:

$u(r)=0$,

$w(r)= \begin{cases}1-r^{7}, & r \leq 1 \\ 0, & r>1\end{cases}$

The streamwise velocity profile (Eq. (4)) is chosen to match approximately the conditions downstream of the nozzle reported in the literature, cf. Liang and Maxworthy [8], to ensure qualitative comparability of the results. To guarantee precisely defined inflow conditions we decided to avoid the superposition of any disturbances at the inflow for accelerating the transition leading to a laminar flow state inside the nozzle due to its relatively short length. All three velocity components at the inflow plane outside of the nozzle are set to zero. The inflow plane outside of the nozzle acts therefore as a solid wall as in the investigations of Liang and Maxworthy [8] and Billant et al. [5], who performed experiments in a large water tank, as well as in those of Facciolo et al. [9] and Oberleithner et al. [4], who attached a plate to the nozzle end. For the case of a nozzle at rest $[5,9,4]$ the position of the solid wall relative to the nozzle end section (or, in other words, the intrusion depth of the nozzle into the tank,) is of minor importance and generally affects the entrainment of ambient fluid only locally (for an extensive discussion see Luginsland [37]). For the rotating nozzle, the intrusion depth may be of importance due to the developing boundary layer at the outer nozzle wall. In the present investigation, the intrusion depth corresponds to the nozzle length and is chosen to $L=5$ in accordance with the experimental setup in [8].

For the rotating nozzle setup, the azimuthal velocity component is defined as solid-body rotation,

$v(r)= \begin{cases}r, & r \leq 1.1 \\ 0, & r>1.1\end{cases}$

Due to the rotation of the nozzle and the no-slip boundary conditions applied, a two-dimensional boundary layer develops at the outer nozzle wall, which is centrifugally unstable according to Leibovich and Stewartson [38]. At the inner nozzle wall a threedimensional boundary layer is present, which is centrifugally stable [38]. The initial vortex core radius [39] of the swirling jet at $t=0$, defined as the region where the fluid is in solid-body rotation, is $r_{c}=1.1$ as a consequence of the nozzle rotation.

For the nozzle at rest, the fluid at the outer nozzle wall is still and no boundary layer develops. At the inner nozzle wall, the streamwise velocity component is zero due to the no-slip boundary condition as for the case with a rotating nozzle. Additionally, the azimuthal velocity component vanishes and the boundary layer is of two-dimensional nature and centrifugally unstable [38]. The azimuthal velocity component at the inflow is defined analytically, similar to the expression used by [18]:

$v(r)= \begin{cases}1.38 \cdot r \cdot \exp \left(-\left(\frac{r}{0.9}\right)^{18}\right), & r \leq 1 \\ 0, & r>1,\end{cases}$

leading to an initial vortex core radius of $r_{c}=0.76$. The azimuthal velocity profile is defined in such a way that its initial maximum $(z=-5, t=0)$ within the nozzle as well as the initial integral swirl numbers $\left(S_{\text {int }}=0.85\right)$ at $t=0$ are identical for both setups. The integral swirl number is defined according to [2] as:

$S_{\text {int }}=\frac{\frac{1}{R} \cdot \int_{0}^{r_{\infty}}\left(\langle w\rangle\langle v\rangle+\left\langle u^{\prime} v^{\prime}\right\rangle\right) r^{2} d r}{\int_{0}^{r_{\infty}}\left(A \cdot r+B \cdot \frac{r^{2}}{2}\right) d r}$,

where

$A=\left(\langle w\rangle^{2}-\frac{\langle v\rangle^{2}}{2}+\left\langle w^{\prime}\right\rangle^{2}-\frac{\left\langle v^{\prime}\right\rangle^{2}+\left\langle u^{\prime}\right\rangle^{2}}{2}\right)$

and

$B=\left(\langle w\rangle \frac{\partial\langle u\rangle}{\partial z}+\langle u\rangle \frac{\partial\langle u\rangle}{\partial r}+\frac{\partial\left\langle w^{\prime} u^{\prime}\right\rangle}{\partial z}\right)$

and \langle\rangle denotes time- and azimuthally-averaged quantities.

As we shall see in the remainder of this paper, the instability mechanism in the nozzle flow is significantly different for the two setups due to the different boundary layers. For the nozzle at rest, helical instabilities are expected to grow within the nozzle wall boundary layer due to shear and centrifugal instability mechanisms [40], and a transition to turbulence takes place already for relatively short nozzles. For the rotating nozzle setup, the boundary layer at the inner nozzle wall is comparably stable and instabilities grow substantially slower in the downstream direction in accordance with [41]. The nozzle flow is expected to stay laminar due to its comparably short length, cf. Imao et al. [42]. The boundary layer at the outer nozzle wall is centrifugally unstable [43] and azimuthal modes are expected to grow in the downstream direction.

Due to the differences in the instability mechanisms for the two setups, the flow conditions downstream of the nozzle where vortex breakdown takes place differ as well. As we will report in Section 4, helical instabilities of different azimuthal mode number grow and saturate at different amplitude levels. These modes interact with the developing conical shear-layer in the region of vortex breakdown and additionally with the growing single-helix type instability believed to dominate the recirculation zone and the inner shear-layer surrounding the vortex-breakdown bubble $[8,4]$.

\section{Comparison of DNS results}

In this section, DNS results for the cases with a rotating nozzle and a nozzle at rest are presented. Mean flow results are compared first, providing insight into the general differences of the flow field and the vortex breakdown structure for the two nozzle setups (Sections 4.1 and 4.2). Effects of the radial density and pressure stratification are discussed. A detailed analysis of the instantaneous flow field (Section 4.3) including baroclinic effects (Section 4.4), of the observed azimuthal modes (Section 4.5) and of the dominating frequencies (Section 4.6) completes the section. 

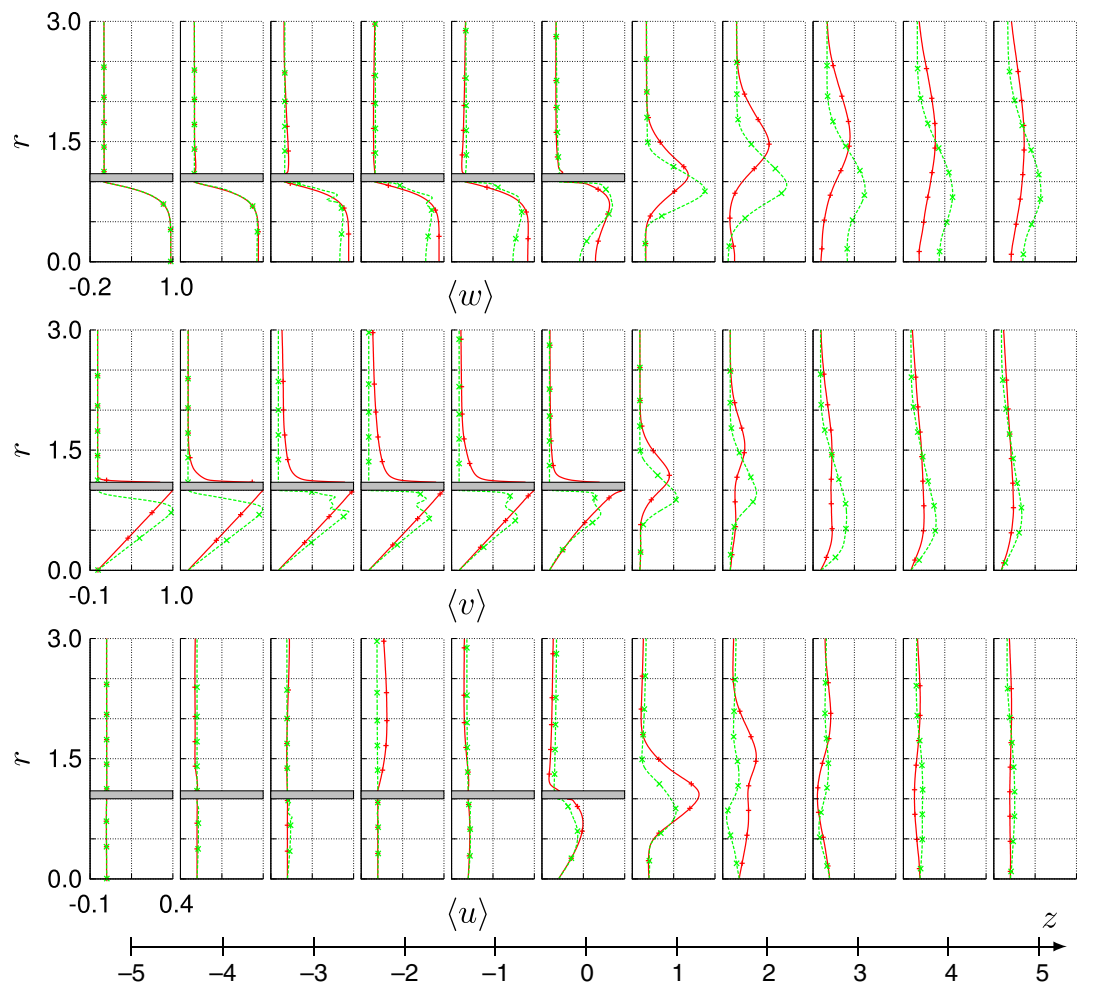

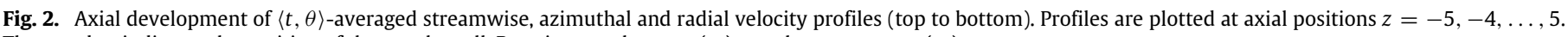
The grey bar indicates the position of the nozzle wall. Rotating nozzle $\_-(+)$, nozzle at rest - - - $(\times)$.

\subsection{Mean flow properties}

Fig. 2 displays the $\langle t, \theta\rangle$-averaged radial distributions of the three velocity components at sequent axial positions. For calculating the time-average data is sampled after 100 dimensionless time units (transition to a quasi-steady flow field) for a time interval of 300 units. Every fifth time-step is used for calculating mean flow properties (15,000 samples), and every 25th time-step is sampled for all other quantities (3000 samples).

For the rotating nozzle, the streamwise velocity component decreases in the streamwise direction due to the upstream effect of vortex breakdown, while maintaining its initial shape within the nozzle. The azimuthal velocity component remains unaltered for the entire nozzle indicating a purely laminar flow as discussed in Facciolo et al. [9]. As reported by Orlandi and Fatica [14] and Imao et al. [42], for a turbulent rotating pipe flow the azimuthal velocity component deviates strongly from an initial solid-body rotation and the streamwise velocity component tends to a parabolic shape being stabilized with increasing swirl. Both effects are not observed in the present investigation. The radial velocity is identically zero for the entire nozzle flow. In the nozzle end section the jet starts to spread radially. At the outer nozzle, a two-dimensional boundary layer is present due to the nozzle in rotation around its axis. The boundary layer thickness increases in the downstream direction with a maximum at $z=-2$ and decreases again further downstream. The local increase of the boundary layer thickness, visible in the azimuthal velocity profiles in Fig. 2(b), is due to the presence of an axisymmetric instability mode $(n=0)$, as discussed in Section 4.5.

For the nozzle at rest, the streamwise velocity component deviates from the initial profile downstream of the inflow plane. The streamwise velocity at the centreline decreases in the downstream direction due to the strong adverse pressure gradient (discussed below). This decrease is in agreement with observations by Vaidya et al. [44], who reported the same effect depending on the swirl number. At $z=-3$, fluid with a high streamwise velocity is shifted radially outwards towards the inner nozzle wall due to the presence of an azimuthal mode of high wave number $(n=$ 12) and the deceleration at the centreline. The azimuthal velocity component is altered due to the developing complex oblique boundary layer, whose thickness decreases significantly in the downstream direction. The initial velocity maximum decreases in favour of a second maximum observed in the vicinity of the nozzle wall. The vortex core size decreases in the downstream direction due to the change in the azimuthal velocity. The streamwise and azimuthal velocity profiles within the nozzle compare qualitatively well with findings reported in Vaidya et al. [44] and Kitoh [45]. The radial velocity component is approximately zero within the entire nozzle except at $z=-3$, where the $n=12$ mode shows its peak amplitude. In the nozzle end section, the radial velocity increases due to the upstream effect of vortex breakdown (jet spreading).

Downstream of the nozzle, the maxima of all three velocity components are located radially further outwards for the rotating nozzle compared to the nozzle at rest. The spreading angle is larger for the rotating nozzle and the maximum radial velocity is higher, indicating a more pronounced vortex breakdown. The momentum transfer in favour of an increased radial velocity at $z=1$ is stronger for the rotating nozzle and comes with a significant decrease of the azimuthal velocity component and a slight deceleration of the fluid within the shear-layer. Contrarily, only a slight increase in the radial momentum and decrease in the azimuthal momentum is observed for the nozzle at rest together with a small acceleration of the fluid inside the shear-layer. The differences observed are due to the azimuthal momentum conservation within the rotating nozzle and the initially larger vortex core compared to the setup with the nozzle at rest. The entrainment of ambient fluid is stronger directly downstream of the nozzle for the rotating nozzle case compared to the nozzle at rest, while it is vice-versa further downstream $(z \geq$ 3 ). The radial and azimuthal velocity components approximately coincide further downstream $(z \geq 5)$ for both setups, while the streamwise velocity profiles still show significant differences. 

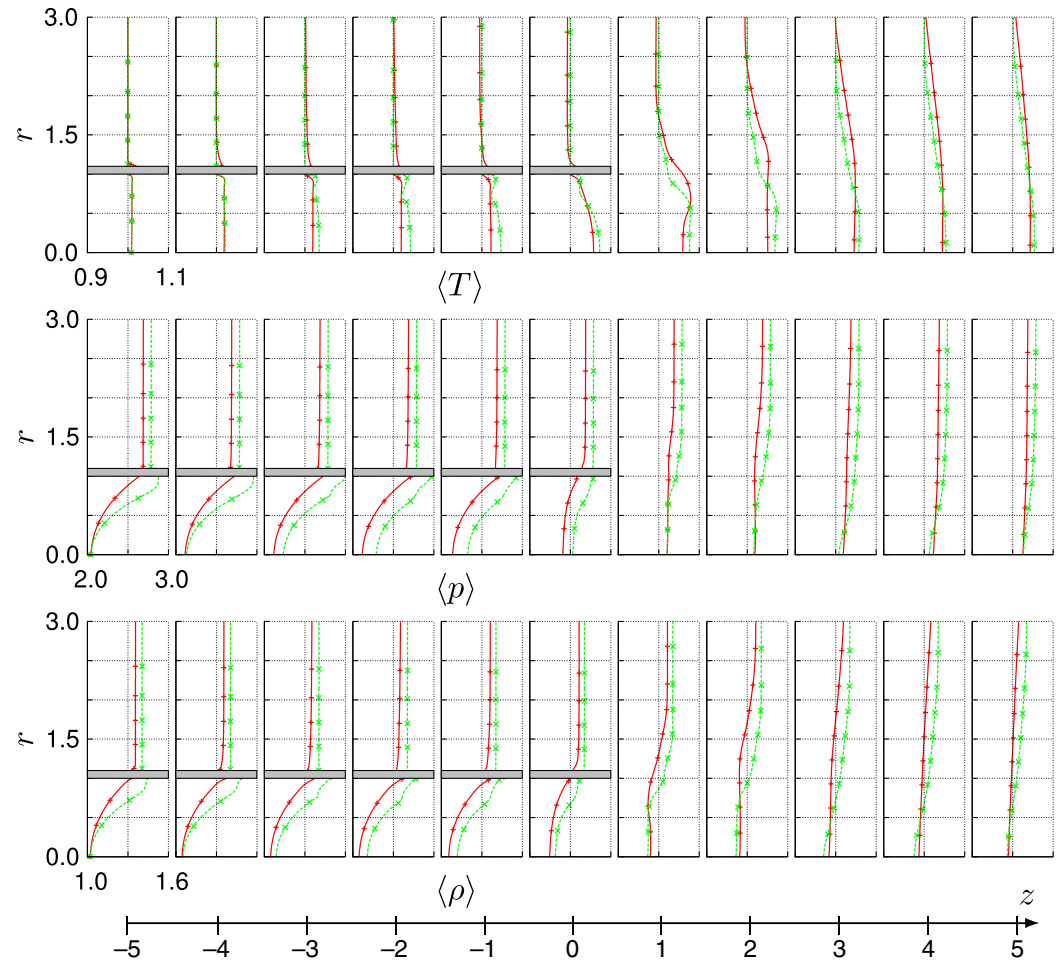

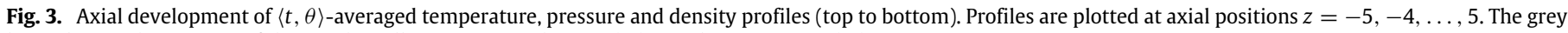
bar indicates the position of the nozzle wall. Rotating nozzle $-(+)$, nozzle at rest $---(\times)$.

The present results compare qualitatively well with experimental findings reported in the literature, cf. Liang and Maxworthy [8] for the rotating nozzle and Oberleithner et al. [4] for the nozzle at rest, respectively. The comparison is restricted to a qualitative analysis here because the initial conditions for the various experiments, the inflow conditions upstream of vortex breakdown (see Section 1 for a discussion) as well as the parameters Reynolds, Mach and swirl number, (Re, $M a, S)$, differ significantly. While all these referred investigations concern the incompressible regime, our investigation focuses on moderately compressible swirling jets at $M a=0.6$ following the previous numerical investigations by $[24,18]$. Comparing our mean flow results [18], we find qualitative agreement as well, but the comparison cannot be conclusive - as for the other references - because of differences in the setups mentioned above, namely in this case the instability mechanisms introduced by modelling the nozzle wall.

The $\langle t, \theta\rangle$-averaged temperature, pressure and density are shown in Fig. 3. For both setups investigated, albeit stronger for the nozzle at rest, there is a temperature increase observed within the nozzle which is due to the deceleration of fluid and a simultaneous increase in the pressure and the density. The flow field inside the nozzle is nearly adiabatic satisfying $\left(0.5 w_{c}^{2}+c_{p} T_{c} \approx\right.$ const.) due to the isothermal nozzle wall. For the nozzle at rest, the temperature increases in the downstream direction in the vicinity of the inner nozzle wall due to the developing complex oblique boundary layer which is explained by dissipative heating [46]. A similar observation, albeit less pronounced, is made for the rotating nozzle within the boundary layer at the outer nozzle wall. Downstream of the nozzle $(z \geq 1)$, the temperature maximum is located in the inner shear-layer of the swirling jet for the rotating nozzle indicating dissipative processes, and in the core region of the jet for the nozzle at rest.

Pressure and density increase more strongly in the radial direction for the nozzle at rest due to the differences in the initial azimuthal velocity distributions (cf. Eqs. (5) and (6)), leading to higher far-field values compared to the rotating nozzle $[18,26]$.
Within the inner wall boundary layer, pressure and density grow more strongly in the downstream direction for the nozzle at rest. This is an effect of the transitional nature of the two-dimensional boundary layer. At the centreline, a similar observation is made due to the deceleration of the fluid and upstream effects of vortex breakdown (for $z \geq-1$ ): the local positive pressure and density gradients in the downstream direction are stronger for the nozzle at rest compared to the rotating nozzle. The pressure distribution for the nozzle at rest is qualitatively comparable to results reported in Kitoh [45]. At the centreline of the swirling jet downstream of the nozzle, pressure and density are nearly identical for both setups, while differences in the far-field $\left(r \rightarrow r_{\infty},-5 \leq z \leq 15\right)$ are still observed due to the far-field sponge imposing different reference solutions for the two setups.

As discussed in earlier publications ([19] and the references therein), the increased core temperature of the flow observed for the nozzle at rest promotes absolute instability and therefore the onset of vortex breakdown. The same effect is attributed to a lower centreline-to-far-field density ratio $\rho_{c} / \rho_{\infty}$ as found for the nozzle at rest compared to the rotating nozzle. Conversely, the lower centreline-to-far-field pressure ratio for the nozzle at rest suppresses vortex breakdown due to a lower expansion rate $p_{c} / p_{\infty}$ compared to the rotating nozzle, counter-acting the temperature and density effects. As we will see in the remainder of this section, the lower expansion rate and the mode selection mechanism acting in the boundary layer at the inner nozzle wall lead to a less pronounced vortex breakdown for the nozzle at rest. Furthermore, baroclinic effects are present for both nozzle configurations which will be discussed below.

The axial development of the $\langle t, \theta\rangle$-averaged streamwise centreline velocity is depicted in Fig. 4. For the rotating nozzle, the flow within the nozzle is characterized by an initial decline in the front part of the nozzle. This is followed by a plateau of constant value $(-4 \leq z \leq-1)$ at whose end a major decrease sets in which is linked to the developing vortex breakdown and its upstream effects inducing a centreline pressure increase. The 


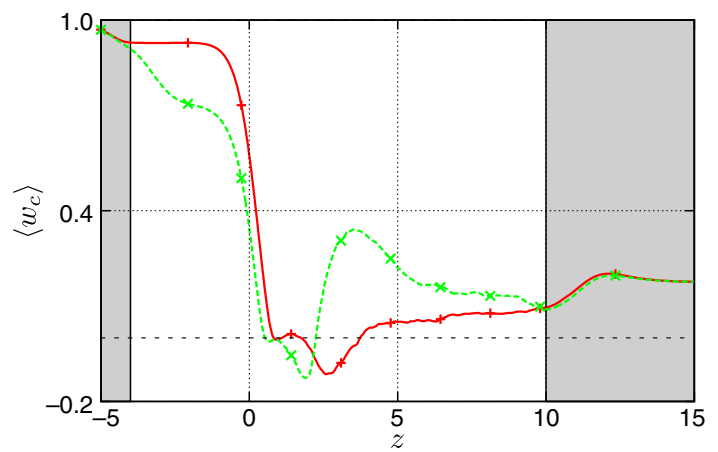

Fig. 4. $\langle t, \theta\rangle$-averaged streamwise velocity at centreline. Rotating nozzle $-(+)$, nozzle at rest - - $-(x)$. Regions where sponges act on the flow field are shaded in grey.

results for the nozzle at rest show a much stronger initial decline of the centreline streamwise velocity within the nozzle as expected (see above). A sharp velocity drop sets in at a downstream position shifted slightly upstream compared to the rotating nozzle case. The upstream shift is approximately identical to the shift of the first stagnation point on the jet axis for the nozzle at rest relative to its location for the rotating nozzle (cf. Fig. 6). The identical negative slope in the streamwise centreline velocity for both nozzle setups indicates comparable upstream effects on the flow within the nozzle. Downstream of the nozzle a distinct breakdown region develops for the two setups. The minimum centreline streamwise velocity is slightly smaller for the nozzle at rest compared to the setup with a rotating nozzle. The streamwise centreline velocity increases again at a position further upstream compared to the rotating nozzle setup, to about $35 \%$ of its initial value. The recovery is due to the relatively moderate spreading of the swirling jet directly downstream of the nozzle lip and the comparably modest streamwise to radial momentum transfer. Downstream of the increase, the centreline velocity decreases in the streamwise direction towards the location where the sponge starts to act on the flow field. For the rotating nozzle, the flow downstream of the recirculation region increases gradually and no pronounced recovery is observed due to the stronger momentum transfer from the streamwise to the radial velocity component.

Fig. 5 shows the streamwise development of the $\langle t, \theta\rangle$-averaged integral swirl number according to Eq. (7). The swirl number is identical for both setups at the inflow plane of the domain $\left(S_{\text {int }}=\right.$ 0.85 ) and increases linearly in the front section of the nozzle to a maximum value of $S_{\text {int }} \approx 1.05$ for the nozzle at rest and $S_{\text {int }} \approx 1.26$ for the rotating nozzle, respectively. The variation is mainly due to the change in the streamwise velocity for $z \leq-4$ for both setups. For $(-4<z \leq-1)$, it is induced by the growth of velocity fluctuations for the rotating nozzle and a combination of those two effects for the nozzle at rest together with a change in the azimuthal velocity component. Further downstream $(z>-1)$, mean flow and velocity fluctuation effects are observed for both setups. For the nozzle at rest, the swirl number decreases in the downstream section of the nozzle to an approximately constant level farther downstream $\left(S_{\text {int }} \approx 0.65\right)$. The integral swirl number decreases as well in the downstream part of the nozzle for the rotating nozzle. It increases substantially in the nozzle end section due to a strong decrease in the second term in the denominator in Eq. (7), $\left(-\langle v\rangle^{2} / 2\right)$, approaching a quasi-constant level further downstream at a much higher level $\left(S_{\text {int }} \approx 0.975\right)$ compared to the nozzle at rest. Spatially averaging the integral swirl number in the vortex breakdown region leads to $S_{\text {int }} \approx 1.05$.

We observe that the integral swirl number $S_{\text {int }}$ varies in the downstream direction for both setups investigated here and that for an identical initial integral swirl number $S_{\text {int }}=0.85$

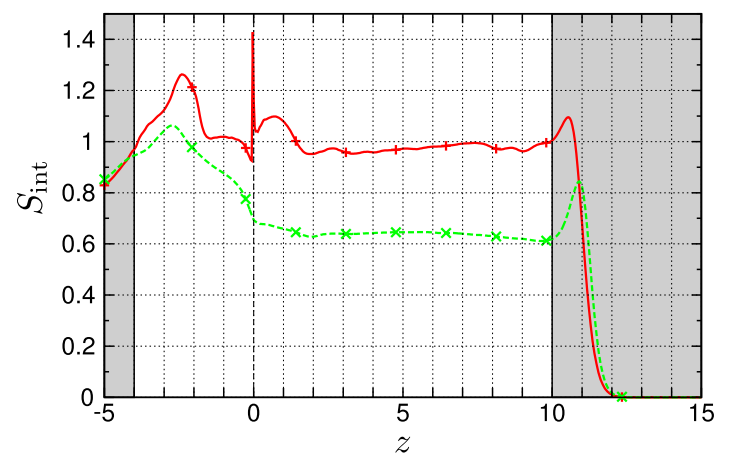

Fig. 5. $\langle t, \theta\rangle$-averaged integral swirl number according to Eq. (7). Rotating nozzle $-(+)$, nozzle at rest - - - $(\times)$. Regions where sponges act on the flow field are shaded in grey.

the $\langle t, \theta\rangle$-averaged swirl number differs at identical streamwise locations. This observation is explained by the angular momentum conservation for the nozzle rotating with the mean flow direction. The angular momentum is conserved in the nozzle flow regime for the rotating nozzle while it is not for the nozzle at rest. A conservation of angular momentum leads to comparably larger terms in the nominator (first term) and denominator (second term of $A$ ), see Eqs. (7) and (8), and therefore to a higher integral swirl number $S_{\text {int }}$. The difference in the approximately constant integral swirl number averaged in the swirling jet flow regime $\left(\Delta S_{\text {int }} \approx 0.325\right)$ is hence an effect of the nozzle flow and due to the differences in the two setups investigated.

\subsection{Vortex breakdown structure}

The recirculation region due to vortex breakdown is significantly smaller for the nozzle at rest, as seen in the streamline plots of the $\langle t, \theta\rangle$-averaged velocity fields (Fig. 6). The location of the minimum streamwise centreline velocity is found in the leeward part of the recirculation zone, which is situated 0.5 radii downstream of the nozzle with a length of 1.75 radii. In the rotating nozzle wall case, the first stagnation point is located 0.8 radii downstream of the nozzle and the length of the recirculation region is approximately 3 radii. The location of the minimum centreline streamwise velocity is shifted by approximately 0.35 radii off the centre of the recirculation bubble in the downstream direction. The internal structure is more complex. A secondary vortical structure is present, introducing two additional stagnation points at the centreline of the swirling jet and an internal shear-layer in the front region of the recirculation zone, in accordance with findings by Faler and Leibovich [47]. It is clearly visible that the radial spreading is much higher for the rotating nozzle compared to the nozzle at rest. The outer jet shear-layer is substantially shifted radially outwards due to the large recirculation bubble, while for the nozzle at rest it is deflected more moderately. The shapes of the recirculation bubbles are a result of the flow conditions upstream, especially of the differences in the momentum balancing for the two setups.

Fig. 7 displays the time-averaged three-dimensional flow field for the two setups. The recirculation bubble for the rotating nozzle is of approximately spherical shape, while for the nozzle at rest the bubble shape is drop-like. These differences, especially in the upstream part, result from differences in the streamwise to radial momentum transfer, which is stronger for the rotating nozzle, as well as from a higher shear found for the nozzle at rest. The streamwise velocity is negative in the leeward part of the recirculation zone for both nozzle configurations, while it is approximately zero (nozzle at rest) or slightly positive (rotating nozzle) in its front part, cf. Fig. 4. For both setups, the fluid counterrotates weakly against the mean flow in the front part of the 


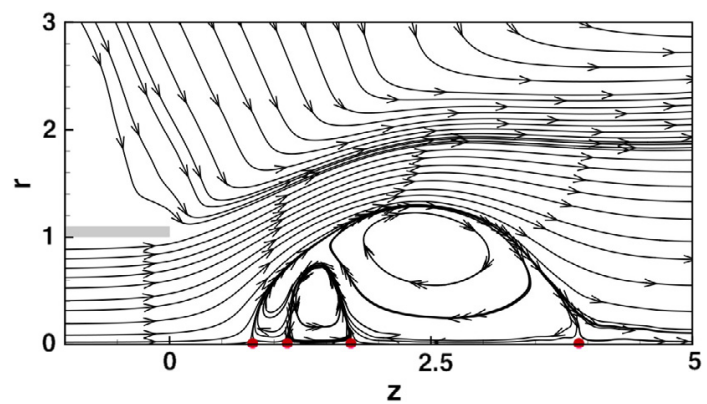

(a) Rotating nozzle.

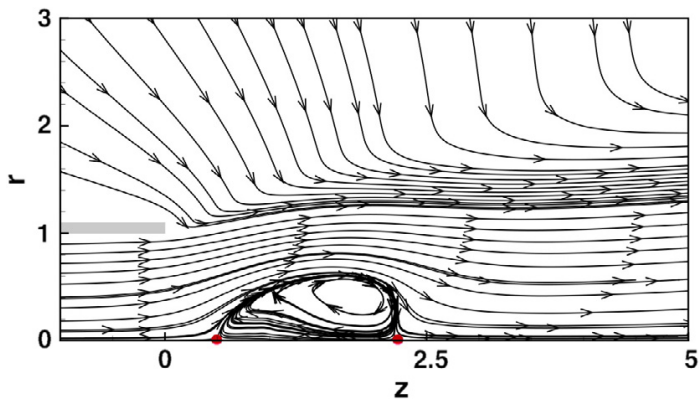

(b) Nozzle at rest.

Fig. 6. Streamlines of the $\langle t, \theta\rangle$-averaged flow field. The grey bar indicates the nozzle wall and the circles the stagnation points at the jet centreline.
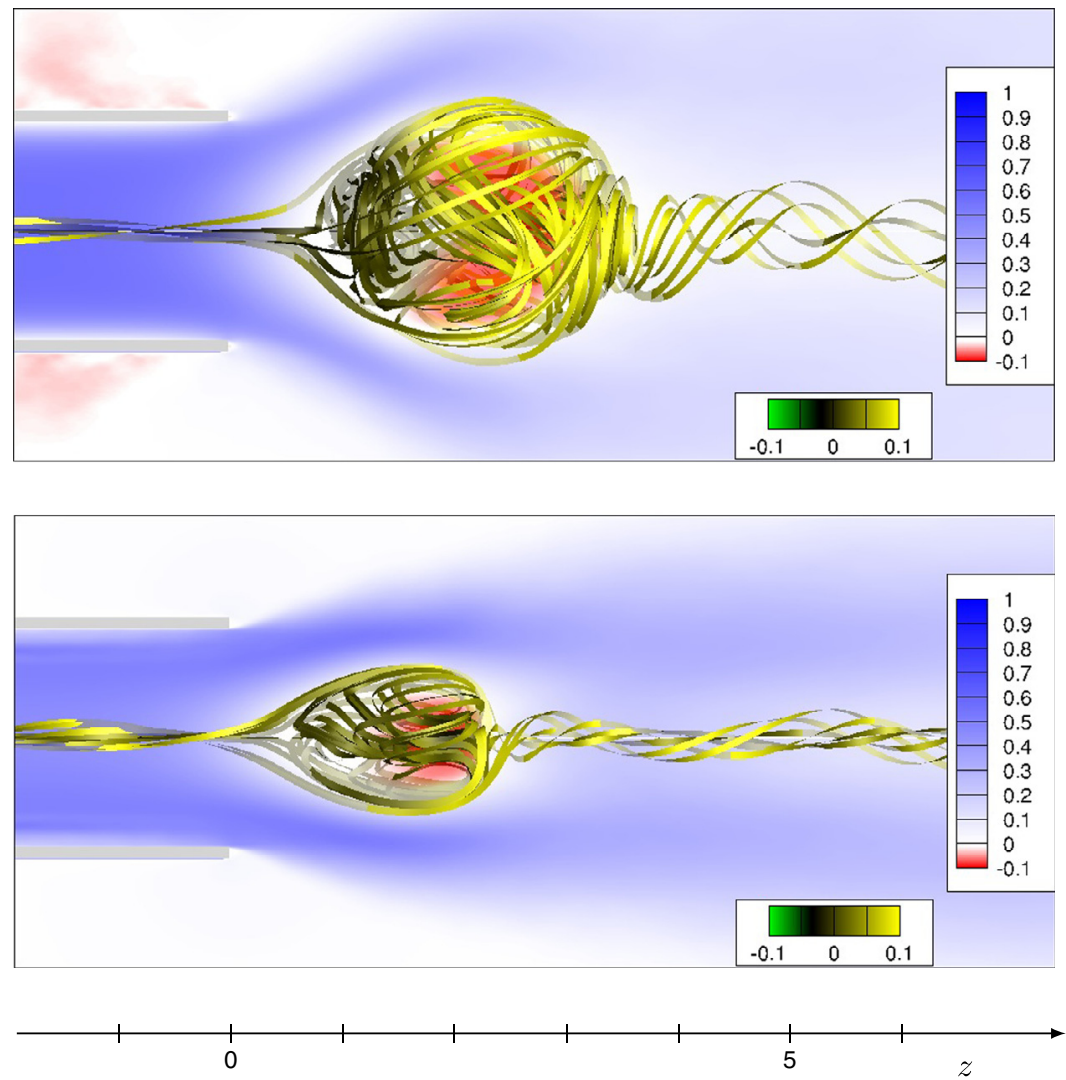

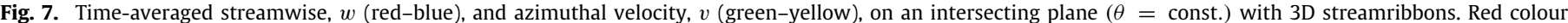

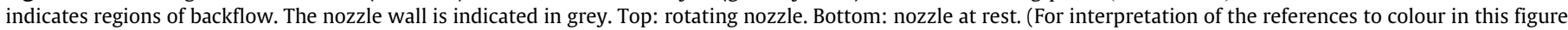
legend, the reader is referred to the web version of this article.)

recirculation zone, as reported in Facciolo and Alfredsson [48] and explained by Reynolds stress effects. In the wake of the vortex breakdown bubble, a time-independent single-helix type instability is observed for both setups, co-winding with the mean flow direction in agreement with observations reported in Sarpkaya [21].

\subsection{Instantaneous flow field}

Figs. 8 and 9 show the instantaneous mass flux field $\rho w$ at sequent downstream positions on $(r, \theta)$-intersection planes at the end of the simulation runs. The instantaneous mass flux $\rho w$ shows a significantly stronger recirculation for the rotating nozzle compared to the nozzle at rest, while it is vice-versa for the maximum value of $\rho w$ (Figs. 8(g) and $9(\mathrm{~g})$ ). This observation is consistent with the differences found in the mean flow for the two different setups as well as for the momentum conservation and transfer, discussed in Section 4.1. The radial stratification of the mass flux is conserved inside the rotating nozzle due to the very low level of fluctuations and the absence of helical instabilities. Strong fluctuations are observed at the outer rotating nozzle wall due to the azimuthal boundary layer present (Fig. 8(a)-(d)). In this region, an axisymmetric mode $n=0$ and a single-helix type mode $n=1$ is observed in agreement with results reported in [49]. The single-helix type instability co-rotates with the mean flow and is of counter-winding type, see Section 4.5 for further discussion.

For the nozzle at rest, a helical instability with high azimuthal wave number $n=12$ is located at the inner nozzle wall within the two-dimensional boundary layer (Fig. 9(b)). This helical mode co-rotates with the mean flow with an opposite winding sense and already decays again upstream of the nozzle end section $(z=0)$. The observation of a co-rotating helical mode with high azimuthal mode number and long axial wavelength is in agreement with findings reported in [50]. Due to the presence of the azimuthal 


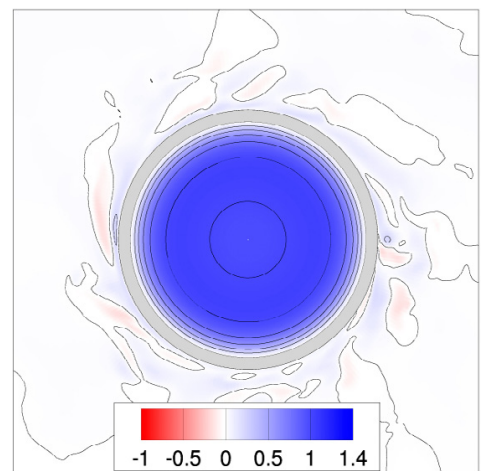

(a) $z=-4$.

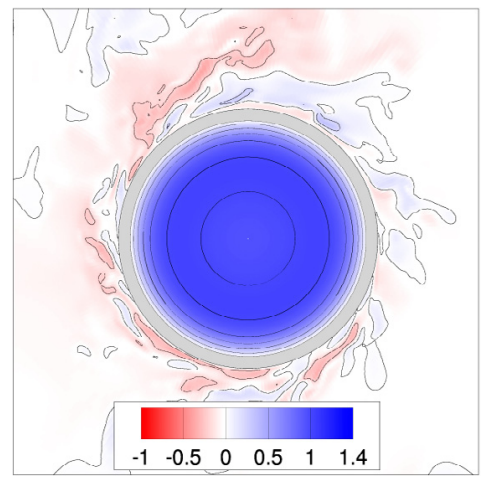

(d) $z=-1$.

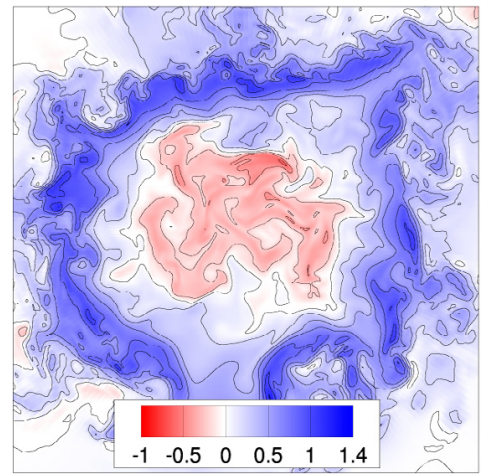

(g) $z=2$.

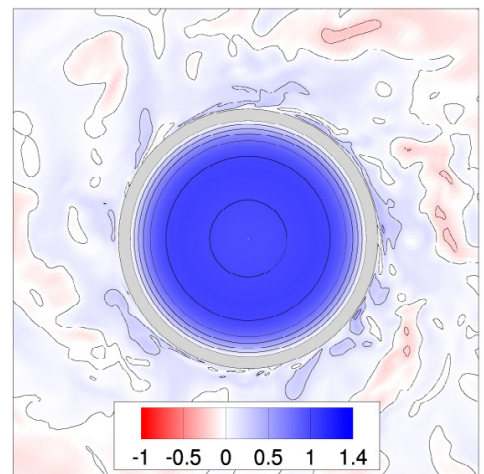

(b) $z=-3$.

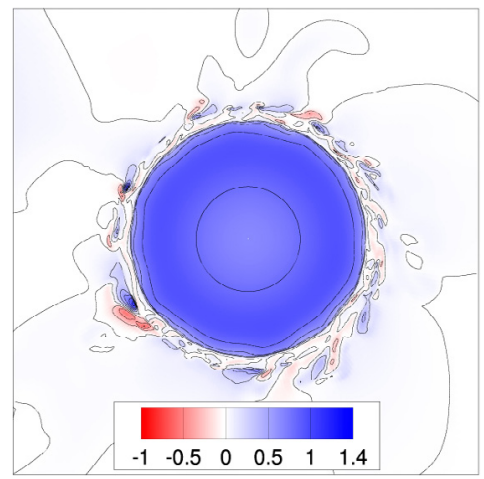

(e) $z=0$.

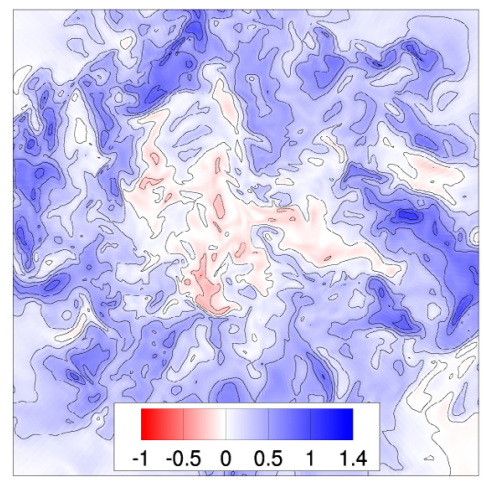

(h) $z=3$.

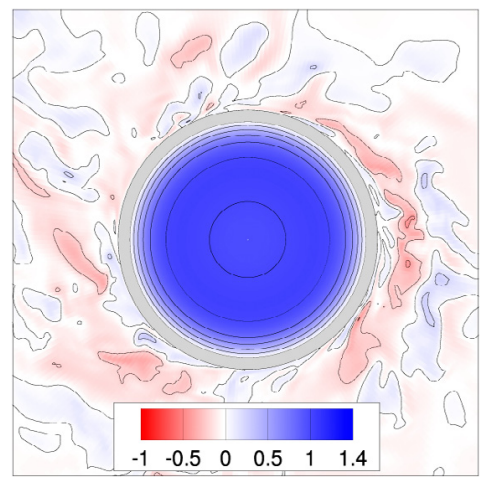

(c) $z=-2$.

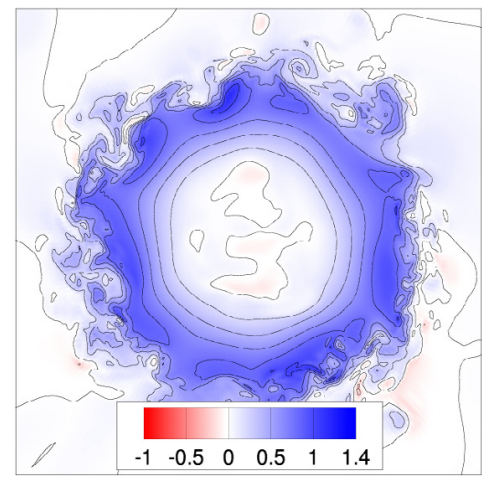

(f) $z=1$.

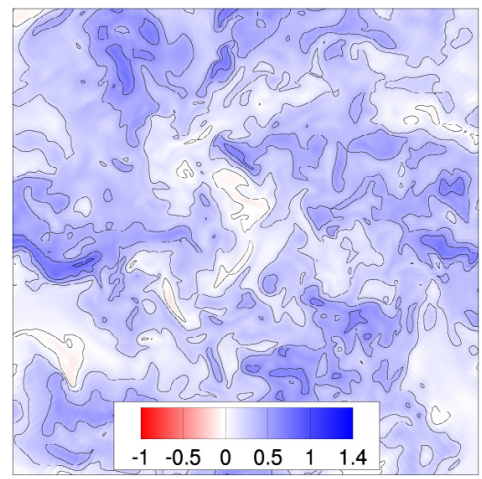

(i) $z=4$.

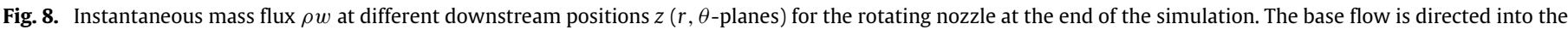
drawing plane and rotates with counter-clockwise orientation. The nozzle wall is indicated in grey.

$n=12$ mode mixing is observed inside the nozzle and the initial mass flux stratification is altered significantly.

Downstream of the nozzle, the swirling jet breaks down more dramatically for the rotating nozzle compared to the nozzle at rest (Figs. 8(f)-(i) and 9(f)-(i)). The region of negative streamwise mass flux is much larger for the rotating nozzle case indicating a larger spatial extent of the vortex breakdown structure. This observation is in correspondence with the initially larger vortex core compared to the nozzle at rest. The spatial structures observed downstream of the nozzle at $1 \leq z \leq 2$ (Figs. 8(f), (g) and 9(f), (g)) inside the shear-layer of the swirling jet are smaller for the nozzle at rest. The reason for this is the transitional nature of the boundary layer at the inner nozzle wall and the growth of high mode number helical instabilities enhancing the mixing process upstream of the swirling jet region. For the rotating nozzle, no small-scale structures are observed inside the nozzle and the helical instabilities entrained into the shear-layer are of large spatial scale. The transition of the jet shear-layer starts to take place downstream of the nozzle lip with the entrainment of low wave number azimuthal instabilities. Therefore, the jet shear-layer is initially dominated by large-scale structures. Further downstream $(z \geq 3)$, the spatial structures are comparably large for both nozzle configurations indicating a transitional process taking place more rapidly for the rotating nozzle.

\subsection{Baroclinic effects and vorticity}

Initially, the mean pressure and density distributions according to Eq. (2) lead to a stratification in the radial direction only. Therefore, the density and the pressure gradients are perfectly aligned in the radial direction at the time of initialization and the baroclinic torque, $\mathbf{B}=(\nabla \rho \times \nabla p) / \rho^{2}$, is zero. The picture changes for the instantaneous flow field at $t>0$ as is discussed in the following. 


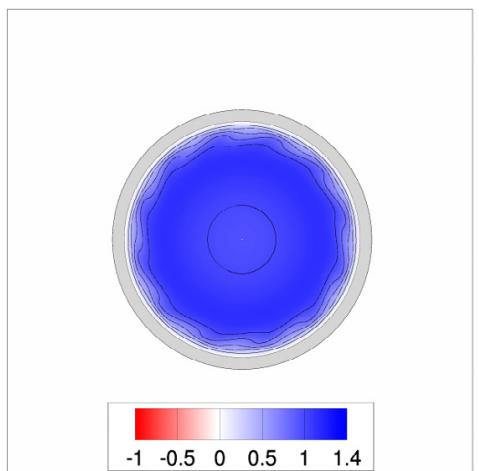

(a) $z=-4$

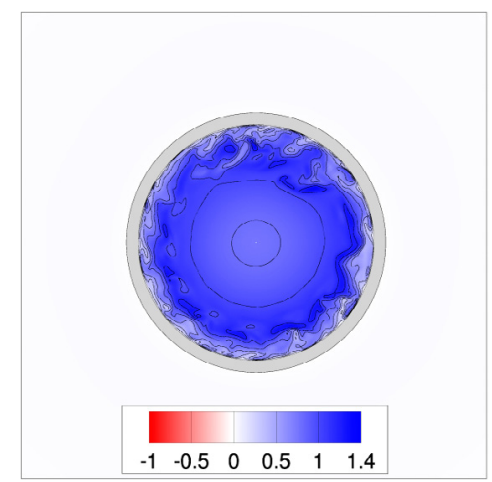

(d) $z=-1$

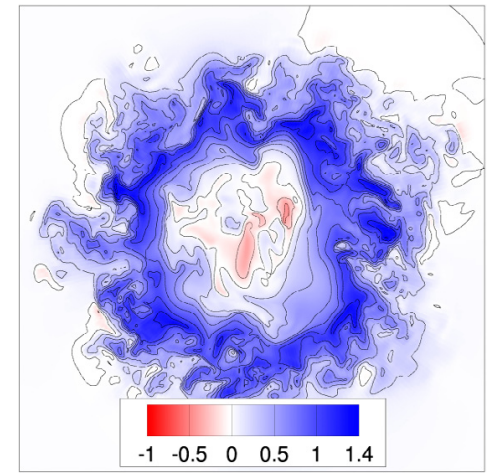

(g) $z=2$.

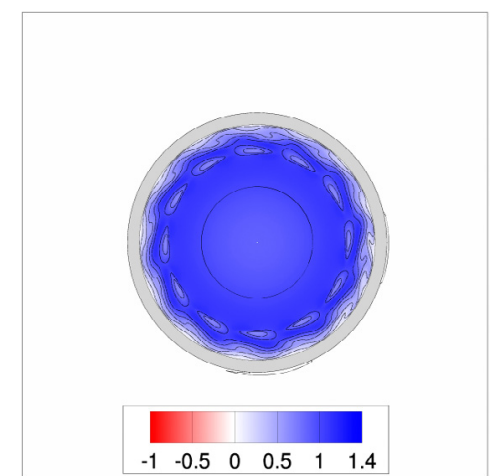

(b) $z=-3$

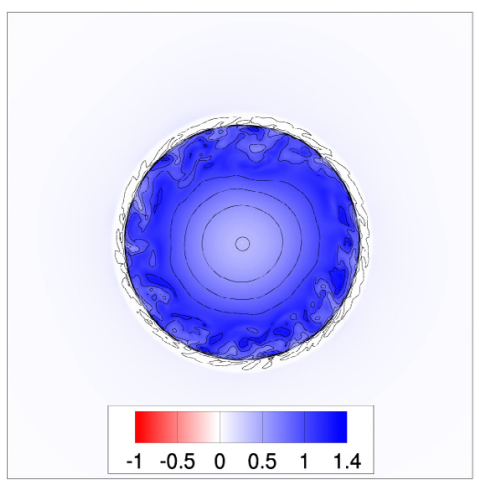

(e) $z=0$.

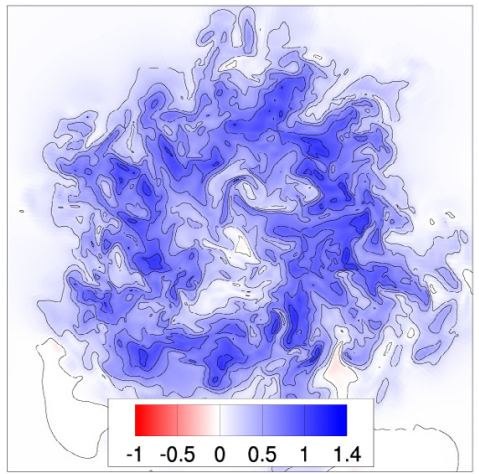

(h) $z=3$.

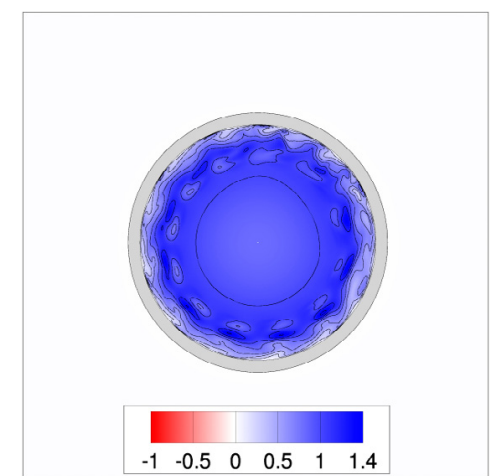

(c) $z=-2$

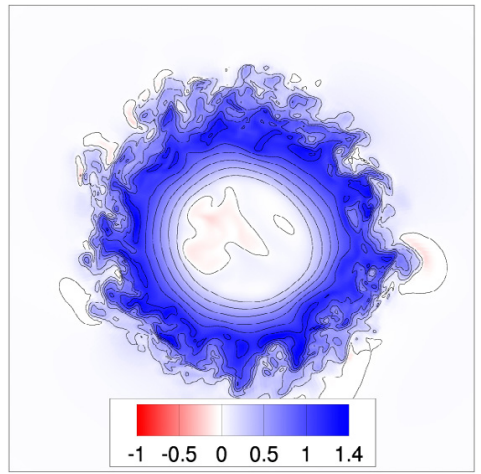

(f) $z=1$.

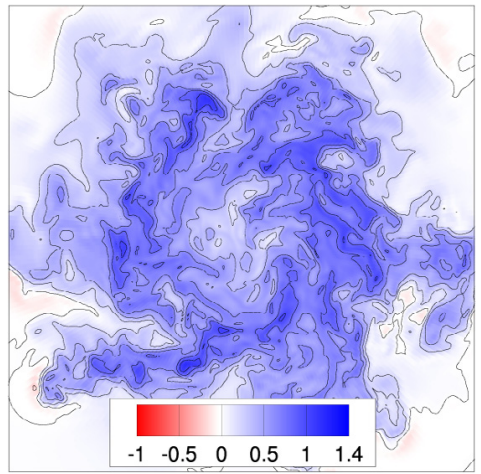

(i) $z=4$.

Fig. 9. Result for the nozzle at rest. For legend see Fig. 8 .

The baroclinic torque is visualized in Figs. 10 and 12 for one instance of time at the end of the simulation runs together with the vorticity magnitude (see also Fig. 11) to get insight into instantaneous effects of the density and pressure stratification. The baroclinic torque is non-zero within the outer shear-layer of the swirling jet for the rotating nozzle as well as for the nozzle at rest with smaller amplitudes observed for the latter. Further downstream $(z \geq 2)$ the baroclinic torque decreases to amplitude levels comparable to those observed in the boundary layer at the outer side of the wall for the rotating nozzle and inside the inner boundary layer for the nozzle at rest, respectively.

In Fig. 11 the instantaneous vorticity field is shown. For both nozzle configurations the vorticity magnitude is high inside the outer jet shear-layer and in the downstream part and the wake of the recirculation region. A decay in the vorticity is observed further downstream for $z \geq 5$ due to diffusive processes, with slightly smaller amplitudes for the rotating nozzle case. High vorticity magnitudes are additionally observed within the boundary layers at the nozzle walls. Directly in front of the stagnation point a large region of negative (azimuthal) vorticity is found for both cases. This is in agreement with Brown and Lopez [51] who identified a physical mechanism driven by the presence of negative vorticity leading to vortex breakdown. In general, the vorticity magnitudes are comparably large for both nozzle configurations.

Comparing Figs. 10-12 one observes that the regions of strong baroclinic torque coincide in general with regions of high vorticity magnitude for both setups investigated. Furthermore, regions of alternating sign in the vorticity distribution coincide approximately with those of the baroclinic torque (see Fig. 12) similar to observations made by [52], but not as clearly distinguishable due to additional mechanisms present, which contribute to the vorticity production, such as strong shear. In our case the baroclinic torque contributes to the vorticity production within the outer shear-layer for both nozzle configurations, as well as within the boundary layer at the inner nozzle wall for the nozzle at rest. Comparing the vorticity field with the field of the baroclinic torque we conclude that 

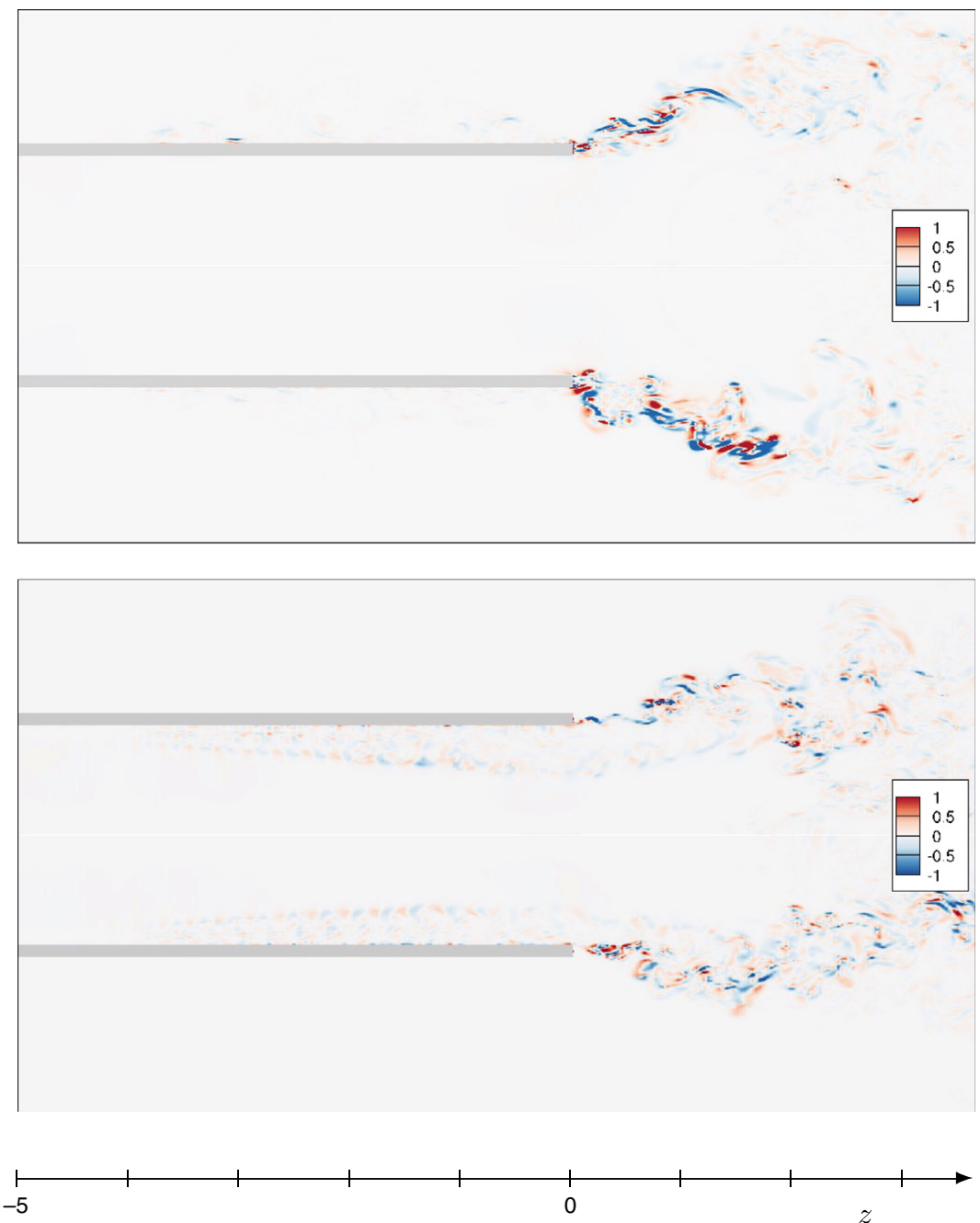

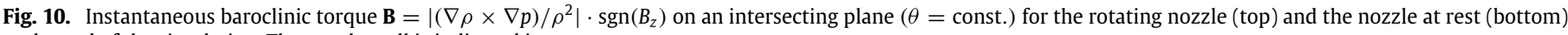
at the end of the simulation. The nozzle wall is indicated in grey.

the baroclinic effects contribute to the production of vorticity, but only in a minor fashion.

This conclusion is confirmed by the budget of the vorticity equation

$$
\begin{aligned}
\frac{\partial \boldsymbol{\omega}}{\partial t}+(\mathbf{u} \cdot \nabla) \boldsymbol{\omega}= & \underbrace{(\boldsymbol{\omega} \cdot \nabla) \mathbf{u}}_{I}-\underbrace{\boldsymbol{\omega}(\nabla \cdot \mathbf{u})}_{I I} \\
& +\underbrace{\frac{1}{\rho^{2}} \nabla \rho \times \nabla p}_{I I I}+\underbrace{\frac{\mu}{\rho} \nabla^{2} \boldsymbol{\omega}}_{I V},
\end{aligned}
$$

where $\omega_{i}$ is the $i$ th component of vorticity, and $\epsilon_{i j k}$ is the LeviCivita symbol. Term (I) represents the vortex stretching, term (II) the volumetric compression, term (III) baroclinic effects and term (IV) viscous diffusion. As displayed exemplarily in Fig. 13, term (III) is significantly smaller compared to the other terms indicating the minor importance of baroclinic effects and the dominating effect of viscous diffusion.

\subsection{Azimuthal mode analysis}

We define the amplitude of the azimuthal modes $n=$ $0, \ldots, 20$ as the time-averaged azimuthally Fourier-transformed instantaneous azimuthal velocity fluctuations

$A_{\theta}(r, z, n)=\left\langle\left|\widehat{v^{\prime}}(r, z, t, n)\right|\right\rangle_{t}$, where $\widehat{()}$ denotes the Fourier transform and ()$^{\prime}$ a fluctuating quantity. The flow downstream of the nozzle is dominated by the single-helix type instability $n=1$ for both setups, see Fig. 14, accompanied by a double-helix instability $n=2$ and the axisymmetric mode $n=0$. The relative dominance of the single-helix type instability is stronger for the nozzle at rest. The maximum value is found one radius further upstream compared to the rotating nozzle, because the recirculation region is located further upstream for the nozzle kept at rest, see Section 4.2. All modes grow exponentially downstream of the nozzle up to comparable saturation amplitude levels for both nozzle setups. The amplitude growth in the downstream direction is stronger for the nozzle at rest due to the stronger shear present in the comparably thinner conical shear-layer as compared to the rotating nozzle. An exception is the axisymmetric mode $n=0$ for the rotating nozzle, whose amplitude is nearly constant downstream of the nozzle dominating the flow for about one radius. For this mode the exponential growth sets in with a delay of about 1.5 radii in the downstream direction. For $z \geq 10$, the outflow sponge starts to act on the flow field leading to a damping of all modes.

In the nozzle flow regime, the mode analysis shows a different picture for the two different setups: for the rotating nozzle helical modes $n=0, \ldots, 20$ grow at the outer nozzle wall saturating at amplitude levels hierarchically ordered by the mode number. The axisymmetric mode $n=0$ dominates the flow together with a co-dominant single-helix type instability, in agreement 

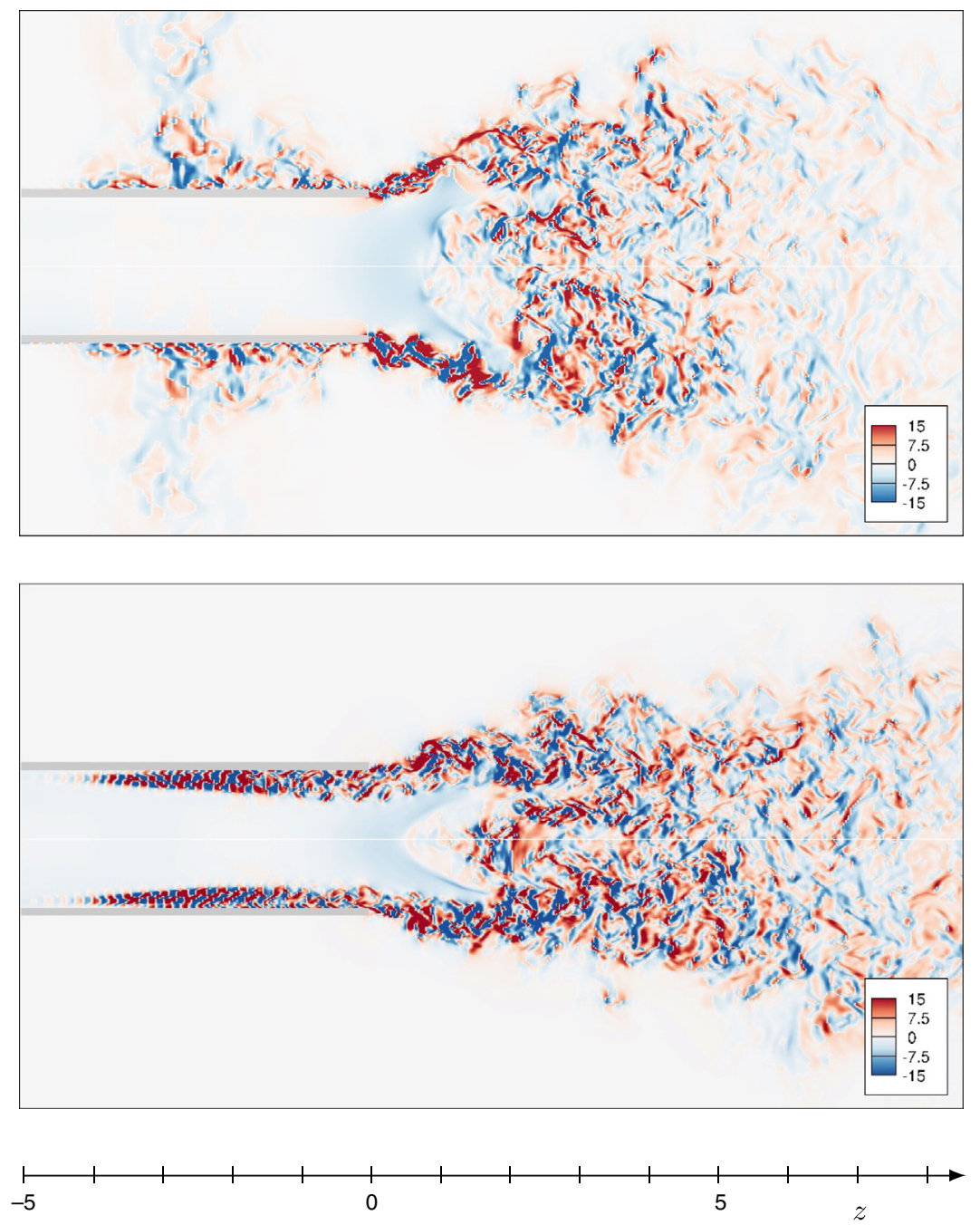

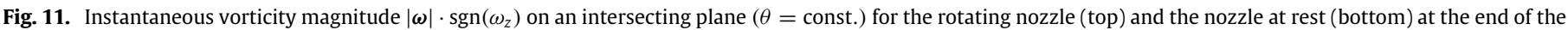
simulation. The nozzle wall is indicated in grey.

with [49]. The downstream growth of the azimuthal boundary layer thickness supports the trend towards low wave number azimuthal modes. Helical modes $n \geq 2$ saturate at the downstream location $z=-2.5$ while the axisymmetric mode and the singlehelix $n=1$ mode reach their amplitude maximum at $z=-2$ and $z=-1.5$, respectively. After reaching a peak value around $z=-3$, the azimuthal amplitudes of all modes decrease again towards the nozzle end section. The decrease is stronger for modes with a high azimuthal wave number due to dissipative processes and a streamwise increase of the boundary layer thickness which supports vortex pairing and an energy transfer to low wave number azimuthal modes.

For the nozzle at rest, the amplitudes of the helical modes $0 \leq n \leq 20$ are in general up to one order of magnitude smaller compared to the rotating nozzle case. The nozzle flow is strongly dominated by a $n=12$ mode together with a co-dominant singlehelix mode $n=1$ and mode $n=13$. The single-helix type instability is found in the centreline region of the nozzle while the azimuthal modes $n=12,13$ are located within the boundary layer at the inner nozzle wall. While the mode amplitude of the singlehelix type instability grows monotonically for the entire nozzle flow, the modes $n=12$ and $n=13$ decay again already within the nozzle. According to spatial linear stability theory [50] the shearmodes $n=12$ and $n=13$ are most unstable and characterized by a rotation sense in the direction of the mean flow as well as by a long axial wavelength. We indeed observed (not shown here, see [22]), that the wave number of the dominant azimuthal mode depends on the swirl intensity. Still, the existence of a helical mode of high azimuthal mode number is a robust pattern of rotating and non-rotating pipe flows, as observed for the latter by Hof et al. [53] and Vaidya et al. [44].

Fig. 15 shows the maximum amplitude of all azimuthal modes $n=0, \ldots, 20$ at each location in $(r, \theta)$-space and the respective mode numbers $n$. For the rotating nozzle flow, the overall maximum amplitude is observed at the outer nozzle wall within the boundary layer, which is centrifugally unstable. Local maxima are located within the inner and outer shear-layer of the swirling jet and in the leeward part of the recirculation zone where the single-helix type instability is found. At the outer nozzle wall, the flow is dominated by an axisymmetric and a single-helix type mode. In the centreline region, the single-helix mode shows the highest amplitude. The breakdown region and the outer shearlayer are governed by azimuthal modes $n=1, \ldots, 3$, the inner shear-layer by the axisymmetric mode $n=0$. At the nozzle lip, azimuthal modes of higher wave number grow and interact with the outer shear-layer of the swirling jet.

The picture for the nozzle at rest is different: high amplitudes are observed in the boundary layer at the inner nozzle wall, where the $n=12$ mode dominates the flow field. As for the rotating nozzle, the centreline region is dominated by the single-helix type instability. Local maxima are located in the inner and outer shear-layer of the swirling jet and in the leeward part of the 


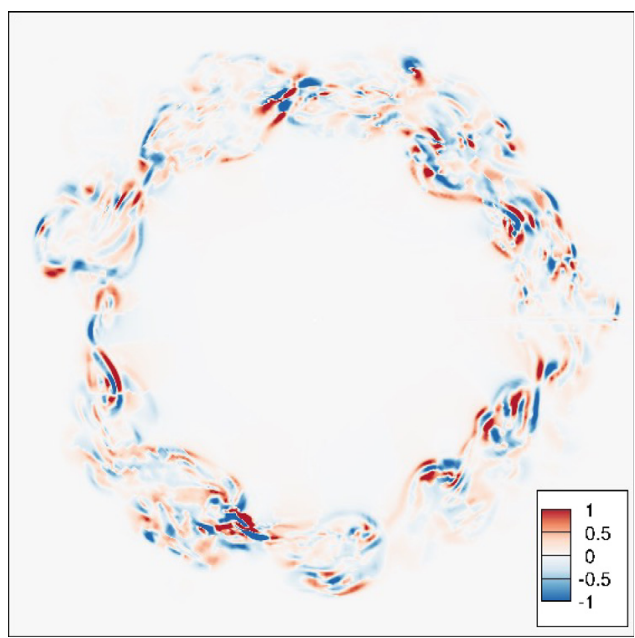

(a) Baroclinic torque.

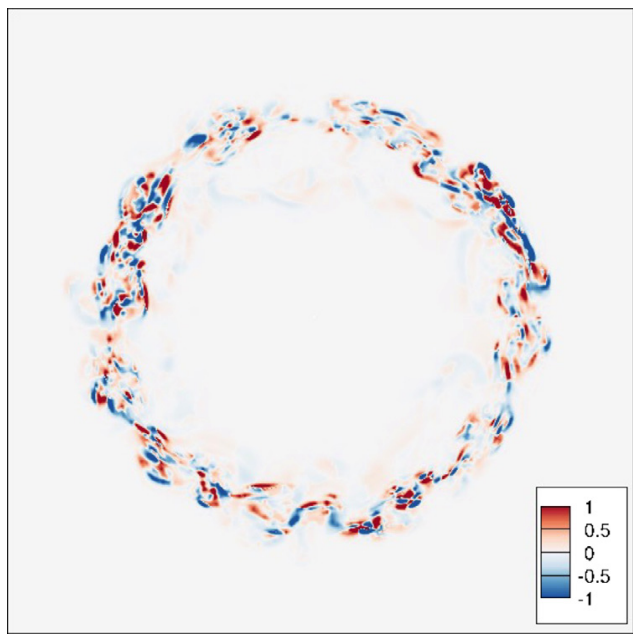

(c) Baroclinic torque.

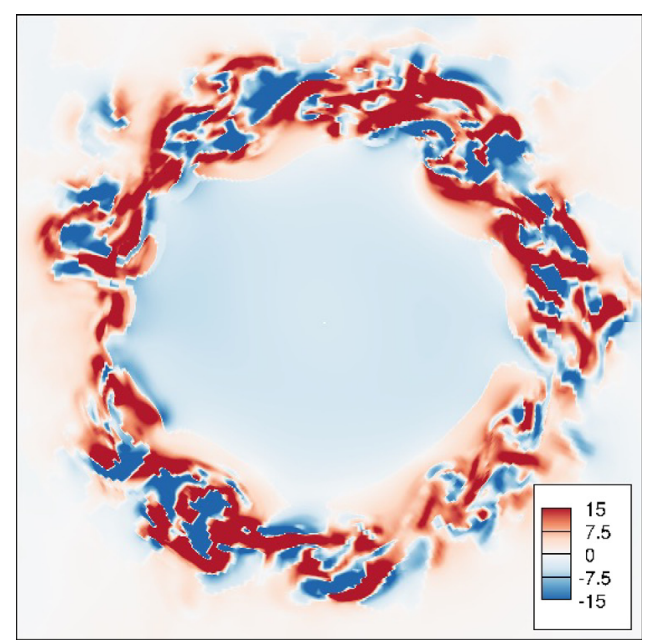

(b) Vorticity.

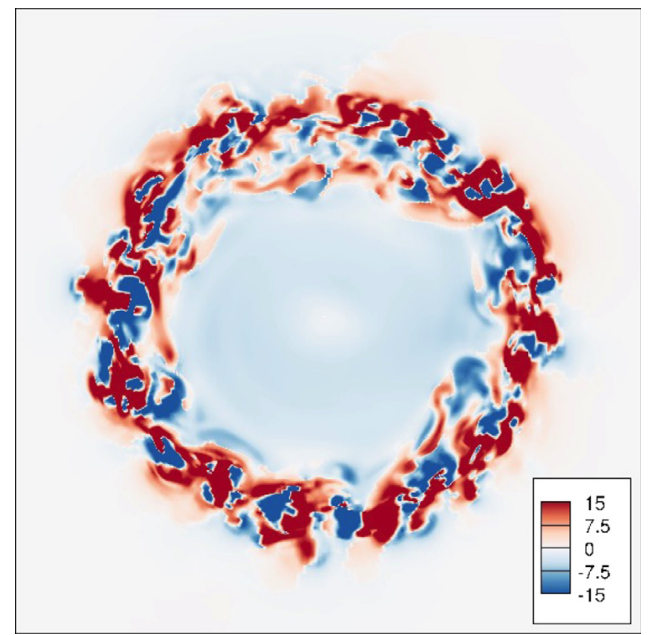

(d) Vorticity.

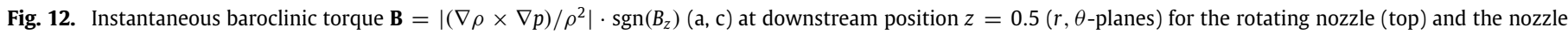

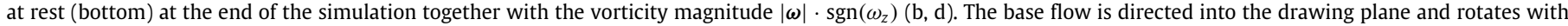
counter-clockwise orientation.

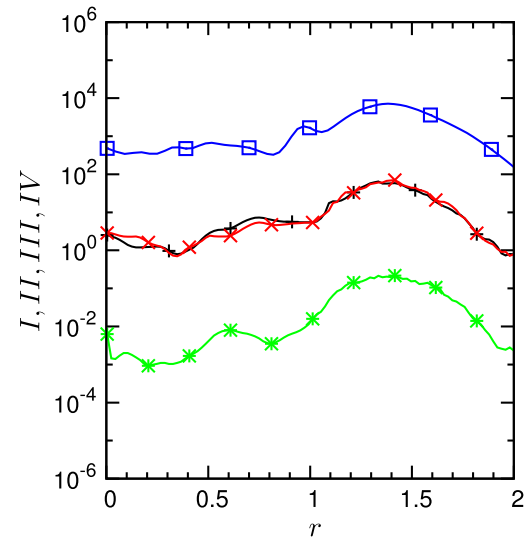

(a) Rotating nozzle.

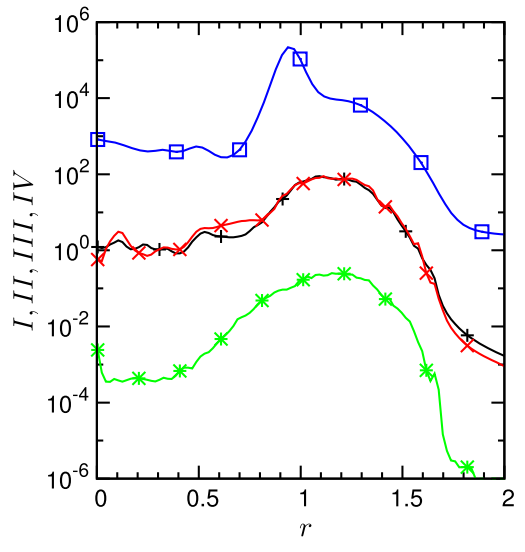

(b) Nozzle at rest.

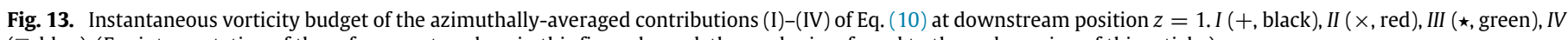
( $\square$, blue). (For interpretation of the references to colour in this figure legend, the reader is referred to the web version of this article.)

recirculation zone, comparable to the rotating nozzle but at higher amplitudes. These higher maximum amplitudes indicate a higher spatial coherence of the azimuthal instabilities (at comparable radially integrated amplitudes, see Fig. 14). A local maximum situated downstream of the vortex breakdown bubble is observed for the nozzle at rest only. In the breakdown region, modes $n=1$ and $n=2$ are observed, while the inner shear-layer is dominated by the axisymmetric mode $n=0$. The outer shear-layer is 


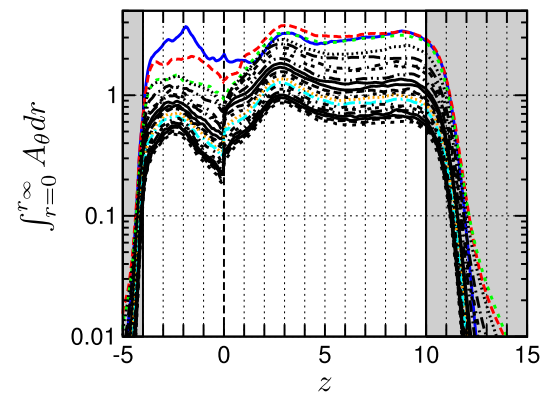

(a) Rotating nozzle.

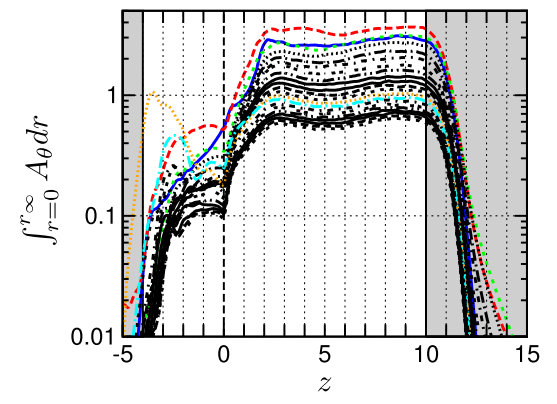

(b) Nozzle at rest.

Fig. 14. Radially integrated mode amplitude. Regions where sponges act on the flow field are shaded in grey. $n=0 \_$(blue), $n=1---($ red), $n=2 \ldots \ldots$ (green), $n=12 \ldots \ldots \ldots$ (orange), $n=13 \ldots$ - . (turquoise), other azimuthal modes in hierarchical order. (For interpretation of the references to colour in this figure legend, the reader is referred to the web version of this article.)

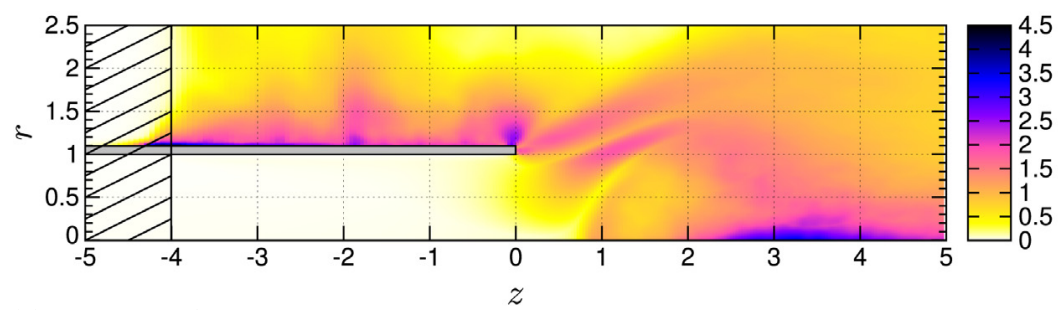

(a) Rotating nozzle.

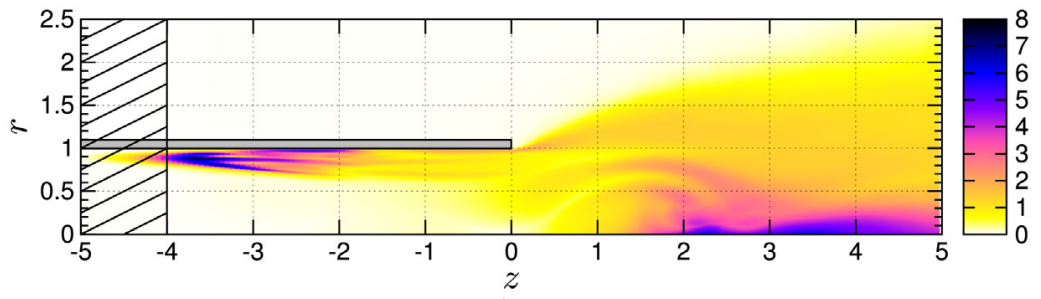

(b) Nozzle at rest.

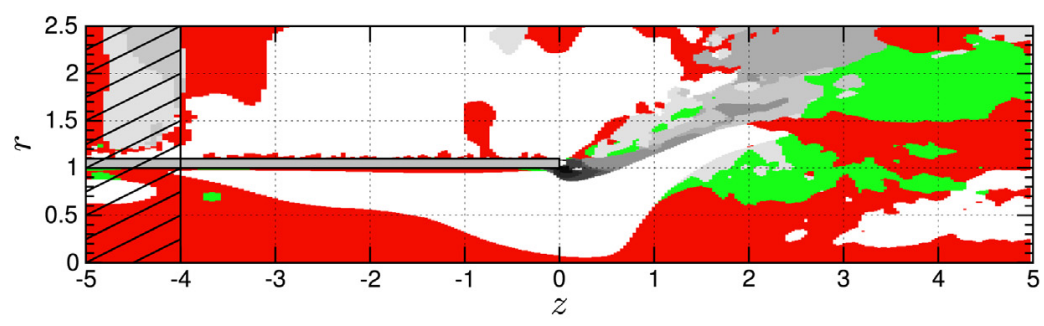

(c) Rotating nozzle.

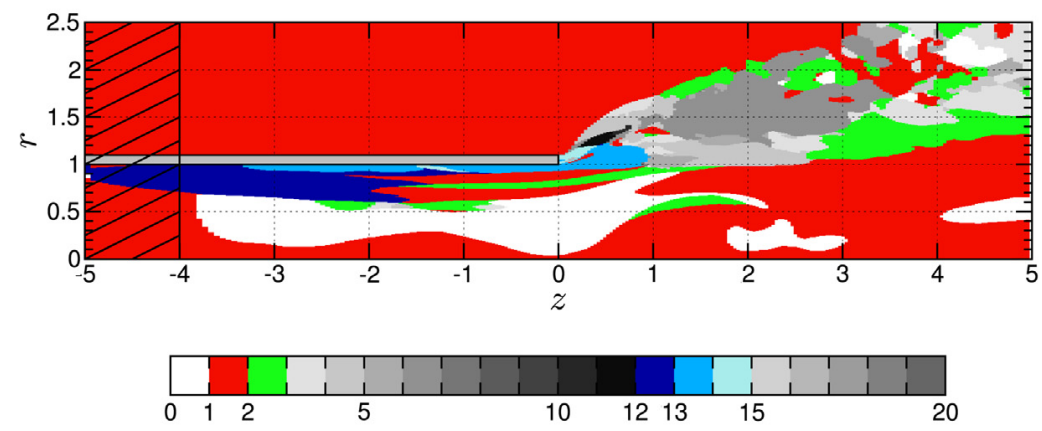

(d) Nozzle at rest.

Fig. 15. (a, b) Maximum amplitude $\max _{n}\left(A_{\theta}(r, z)\right)$ of all azimuthal modes $n$ and $(c, d)$ azimuthal modes $n$ associated with the $\operatorname{maximum}$ amplitude max $_{n}\left(A_{\theta}(r, z)\right)$. $n=0$ (white), $n=1$ (red), $n=2$ (green), $n=12$ (dark blue), $n=13$ (light blue), other azimuthal modes indicated in grey. The inflow sponge region is hatched, the nozzle wall is indicated as grey bar. (For interpretation of the references to colour in this figure legend, the reader is referred to the web version of this article.) 
Table 1

Overall dominant Strouhal number $\left(S t=f^{\circ} \cdot R^{\circ} / w_{c}^{\circ}\right)$ with amplitude and location.

\begin{tabular}{llll}
\hline Nozzle setup & $S t_{\text {dom }}$ & $A\left(S t_{\text {dom }}\right)$ & $(r, \theta, z)\left(S t_{\text {dom }}\right)$ \\
\hline Rotating & 0.005 & 0.428 & $(1.223,0,2.519)$ \\
At rest & 0.103 & 1.331 & $(0.695,0,1.613)$ \\
\hline
\end{tabular}

governed by the $n=13$ mode already observed in the nozzle boundary layer together with modes $n=1$ and $n=2$ and other modes of high azimuthal wave number. The slower spatial growth rate of the $n=13$ mode compared to the $n=12$ mode dominating the boundary layer inside the nozzle was predicted by spatial linear stability analysis [50]. The nozzle lip does not seem to have a strong influence on the mode selection for the nozzle at rest. The modes found in the boundary layer inside the nozzle are observed in the outer shear-layer as well and the flow does not change significantly in the vicinity of the nozzle lip. Further downstream, modes $n=1$ and $n=2$ are found in the outer shear-layer of the swirling jet.

\subsection{Frequency analysis}

The maximum Fourier-amplitudes of the streamwise velocity fluctuations transformed in time according to

$A\left(S t_{\mathrm{dom}}\right)=\max _{S t}\left|\widehat{w^{\prime}}(r, \theta, z, S t)\right|$

are shown at each location in space for both setups in Fig. 16. For the nozzle at rest, maxima are located within the boundary layer at the inner nozzle wall and in the inner shear-layer of the swirling jet (overall maximum). For the rotating nozzle, the maximum amplitude is about three times smaller compared to the nozzle at rest indicating a weaker temporal coherence of the single-helix mode. Local maxima are observed at the outer nozzle wall, in the jet shear-layers and at the centreline within the recirculation zone. The overall maximum is found in the leeward part of the inner shear-layer of the swirling jet in accordance with results for the nozzle at rest.

The Strouhal numbers corresponding to the maximum amplitudes are substantially different for the two setups, see Fig. 16 and Table 1 . This is not surprising in view of the different underlying instability mechanisms governing the flow upstream of the vortex breakdown in the nozzle flow regime. The Strouhal numbers vary significantly depending on the spatial location and the associated azimuthal mode, see Fig. 15, although generally speaking, the Strouhal numbers observed in the flow field for the rotating nozzle cover the same range as for the nozzle at rest. For both setups, the Strouhal number distribution in the outer shear-layer is highly heterogeneous due to the competition and interaction of several helical instabilities. The strongest coherent fluctuation is found in the shear-layer of the vortex breakdown bubble, both for the rotating nozzle and for the nozzle at rest, although the prevailing dominant frequencies strongly differ. We have observed (not reported here, see [22]) that the dominant Strouhal number increases significantly with the swirl intensity. This increase sets in for comparably higher swirl intensities in the case of a rotating nozzle. Therefore, the dominating Strouhal number found in the present study and associated with the maximum Fourier-amplitude observed in the inner shear-layer of the vortex breakdown bubble is significantly smaller than for the nozzle at rest.

\section{Summary, discussion and conclusions}

Vortex breakdown of swirling jet flows in the compressible regime at Reynolds number $R e=5000$ and Mach number $M a=0.6$ was investigated by means of Direct Numerical Simulation (DNS). A nozzle was included in the computational domain, modelled as an isothermal wall. The nozzle was either in rotation with the mean flow or kept at rest. We discussed the main differences in the flow field and especially in the instability mechanisms associated with the two nozzle configurations. The integral swirl number $S_{\text {int }}=0.85$ at the inflow was identical for both setups investigated.

For a rotating nozzle wall, the vortex breakdown of the swirling jet is stronger. The recirculation zone is bigger in the streamwise and the radial directions and the spreading angle is larger. The first stagnation point on the jet centreline is located further downstream compared to the nozzle at rest, as is the second stagnation point. A secondary vortical structure is observed in the front part of the recirculation zone, introducing an additional shear-layer and two stagnation points. The minimum streamwise centreline velocity is slightly larger than for the nozzle at rest. The differences described are linked to the higher integral swirl number observed in the vortex breakdown region for the rotating nozzle. However, the changes in the flow configuration cannot be explained exclusively by swirl number effects: independent of the integral swirl number, differences are observed due to the different underlying instability mechanisms observed in the nozzle flow [22].

Baroclinic effects on the vorticity production were investigated and found to be of minor importance for both nozzle configurations investigated in the present study. The strongest effects of the baroclinic torque were observed inside the outer conical shearlayer, and weaker, in the boundary layers at the inner and outer nozzle walls contributing to a slightly increased vorticity.

The azimuthal mode selection in the nozzle flow differs significantly between the two setups, both in the type of helical instabilities selected as well as in the amplitude level. For the rotating nozzle wall, a boundary layer develops at the outer nozzle wall, which is centrifugally unstable to the low wave number azimuthal modes $n=0$ and $n=1$. The nozzle flow is laminar except for some upstream effects of the vortex breakdown. The nozzle at rest leads to an unstable boundary layer at the inner nozzle wall, where a $n=12$ azimuthal mode grows together with a single-helix mode $n=1$ and a mode of high azimuthal wave number $n=13$. The amplitudes of the azimuthal modes are generally higher for the rotating nozzle. The maximum amplitude is observed within the boundary layer at the outer nozzle wall for the rotating nozzle, while it is located in the boundary layer at the inner nozzle wall for the nozzle at rest. Downstream of the nozzle, the amplitudes are comparably high for both nozzle setups. For both setups, the jet flow is governed by a single-helix type instability $(n=1)$ accompanied by an axisymmetric mode $(n=0)$ together with a double-helix mode $(n=2)$.

A temporal frequency analysis reveals that the maximum temporal Fourier-amplitudes are observed in the nozzle boundary layer and in the inner shear-layer of the swirling jet for the nozzle at rest. The rotating nozzle case shows three maxima at the jet centreline and in the inner and outer shear-layer of the swirling jet. The dominant Strouhal number and the associated Fourieramplitude is higher for the nozzle at rest.

The present study bridges the gap between investigations of swirling jet flows reported in the literature utilizing rotating nozzle devices [8,9] and nozzles at rest [5,10,4,11]. A numerical framework is used that allows to study both nozzle configurations with a minimum of other setup differences. Furthermore, the present study goes beyond recent experimental and numerical investigations of swirling jet flows undergoing vortex breakdown in that it documents both the nozzle flow and the swirling jet flow at the same time. The present results give strong evidence of the significant influence of the nozzle wall motion (rotating or at rest) on the vortex breakdown behaviour of the swirling jet. The boundary layers developing at the inner (nozzle at rest) and outer (rotating nozzle) nozzle wall strongly influence the vortex 


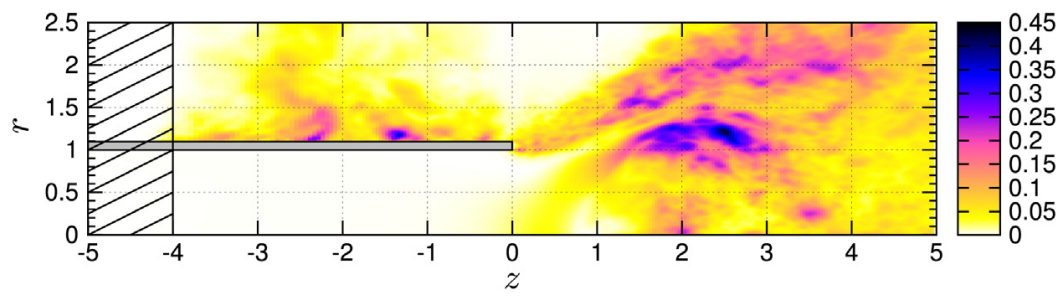

(a) Rotating nozzle.

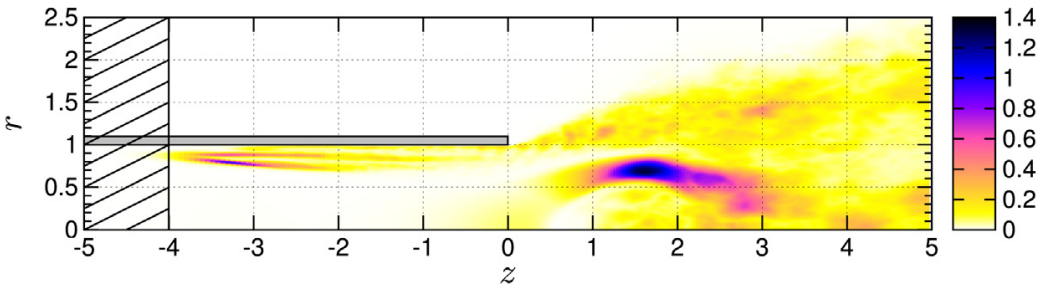

(b) Nozzle at rest.

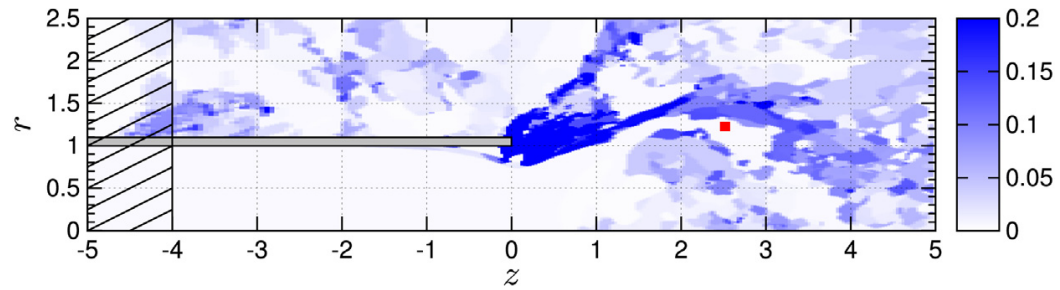

(c) Rotating nozzle.

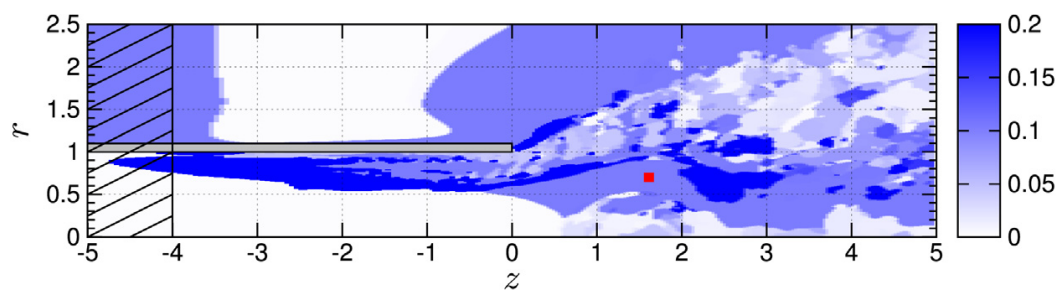

(d) Nozzle at rest.

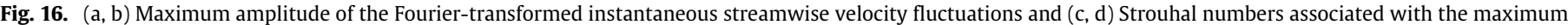

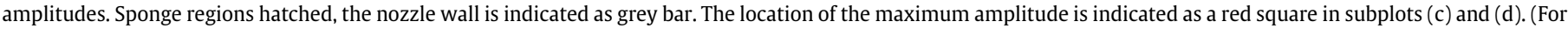
interpretation of the references to colour in this figure legend, the reader is referred to the web version of this article.)

breakdown configuration. Swirling jets with an initially identical swirl number at the inflow show significant variations in the size and shape of the vortex breakdown region as well as in its intensity. These differences in the results are observed over a wide range of the swirl intensity, reaching from the pre-breakdown regime to highly swirling jet flows [22]. Consequently, the present observations re-emphasize that comparisons of results reported in the literature should be made with care, especially when the flow upstream of vortex breakdown is not documented and therefore largely unknown.

The conclusion is drawn that the changes observed in the nozzle (laminar vs. transitional flow, azimuthal momentum conservation vs. momentum transfer), the different types of boundary layers developing in the downstream direction and, following from this, the variation in the mode selection upstream of vortex breakdown are responsible for the substantially different flow fields observed for the swirling jet. Still, we would like to point out that, apart from the differences described in the last section, the overall instability mechanism leading to vortex breakdown as well as the mode selection (single-helix type $n=1$ instability) in the recirculation region is the same for both setups investigated. This gives strong evidence of the basic robustness of the mechanisms leading to vortex breakdown and provides a reasonable fundament to compare studies of swirling jet flows emanating from rotating or fixed nozzles.

The present study clarifies the important role of the nozzle flow for the vortex breakdown in the swirling jet flows emanating from the nozzle. It therefore strongly suggests to include a documentation of the nozzle flow in future studies of swirling jet flows, in order to provide a more complete picture of the entire flow field and to allow for a more meaningful comparison of different studies.

\section{Acknowledgements}

The present work was funded by the ETH research grant ETH-18 08-1 and supported by a grant from the Swiss National Supercomputing Centre (CSCS), Lugano, under project ID s52. The authors would like to thank Y. Reinhardt for remarks on the manuscript and helpful discussions as well as the reviewers for recommendations that helped to improve the manuscript. 


\section{References}

[1] D. Peckham, S. Atkinson, Preliminary results of low speed wind tunnel tests on a gothic wing of aspect ratio 1.0. Technical Report British Aeronaut. Res. Council Aero. 2504, 1957.

[2] N. Chigier, A. Chervinsky, Aerodynamic study of turbulent burning free jets with swirl, in: Symposium (International) on Combustion, Vol. 11 (1) 1967, pp. 489-499.

[3] O.R. Burggraf, M.R. Foster, Continuation or breakdown in tornado-like vortices, J. Fluid Mech. 80 (1977) 685-705.

[4] K. Oberleithner, M. Sieber, C.N. Nayeri, C.O. Paschereit, C. Petz, H.C. Hege, B.R. Noack, I. Wygnanski, Three-dimensional coherent structures in a swirling jet undergoing vortex breakdown: stability analysis and empirical mode construction, J. Fluid Mech. 679 (2011) 383-414.

[5] P. Billant, J.M. Chomaz, P. Huerre, Experimental study of vortex breakdown in swirling jets, J. Fluid Mech. 376 (1998) 183-219.

[6] F. Gallaire, J.M. Chomaz, Mode selection in swirling jet experiments: a linear stability analysis, J. Fluid Mech. 494 (2003) 223-253.

[7] O. Lucca-Negro, T. O’Doherty, Vortex breakdown: a review, Prog. Energy Combust. 27 (4) (2001) 431-481.

[8] H. Liang, T. Maxworthy, An experimental investigation of swirling jets, J. Fluid Mech. 525 (2005) 115-159.

[9] L. Facciolo, N. Tillmark, A. Talamellil, P.H. Alfredsson, A study of swirling turbulent pipe and jet flows, Phys. Fluids 19 (2007) 035105.

[10] F. Gallaire, S. Rott, J.M. Chomaz, Experimental study of a free and forced swirling jet, Phys. Fluids 16 (2004) 2907-2917.

[11] B. Leclaire, L. Jacquin, On the generation of swirling jets: high-Reynoldsnumber rotating flow in a pipe with a final contraction, J. Fluid Mech. 692 (2011) 78-111.

[12] H. Liang, T. Maxworthy, Experimental investigations of a swirling jet in both stationary and rotating surroundings, Exp. Fluids 45 (2) (2008) 283-293.

[13] P. Huerre, P.A. Monkewitz, Local and global instabilities in spatially developing flows, Annu. Rev. Fluid Mech. 22 (1) (1990) 473-537.

[14] P. Orlandi, M. Fatica, Direct simulations of turbulent flow in a pipe rotating about its axis, J. Fluid Mech. 343 (1997) 43-72.

[15] P.G. Saffman, Vortex Dynamics, in: Cambridge Monographs on Mechanics \& Applied Mathematics, Cambridge University Press, 1995.

[16] T.B. Benjamin, Theory of the vortex breakdown phenomenon, J. Fluid Mech. 14 (4) (1962) 593-629.

[17] R. Melville, The role of compressibility in free vortex breakdown, AIAA Paper (96-2075), 1996, pp. 1-16.

[18] S.B. Müller, L. Kleiser, Large-eddy simulation of vortex breakdown in compressible swirling jet flow, Comput. \& Fluids 37 (7) (2008) 844-856.

[19] T. Luginsland, L. Kleiser, Mach Number Influence on Vortex Breakdown in Compressible, Subsonic Swirling Nozzle-Jet Flows, in: ERCOFTAC Series, vol. 20, Springer, Cham, 2015.

[20] M.A. Herrada, M. Perez-Saborid, A. Barrero, Vortex breakdown in compressible flows in pipes, Phys. Fluids 15 (2003) 2208-2218.

[21] T. Sarpkaya, On stationary and travelling vortex breakdowns, J. Fluid Mech. 45 (3) (1971) 545-559.

[22] T. Luginsland, Numerical investigation of vortex breakdown in compressible, swirling nozzle-jet flows (PhD thesis), ETH Dissertation, Zurich, 2014.

[23] Z. Rusak, J.H. Lee, On the stability of a compressible axisymmetric rotating flow in a pipe, J. Fluid Mech. 501 (2004) 25-42.

[24] S.B. Müller, Numerical investigations of compressible turbulent swirling jet flows (PhD thesis), ETH Dissertation, Zurich, 2007.

[25] S. Bühler, Numerical simulation of laminar and turbulent nozzle-jet flows and their sound (PhD thesis), ETH Dissertation, Zurich, 2014.

[26] T. Luginsland, L. Kleiser, Effects of boundary conditions on vortex breakdown in compressible swirling jet flow simulations, Comput. \& Fluids 109 (C) (2015) $72-84$.
[27] J. Delery, Aspects of vortex breakdown, Prog. Aerosp. Sci. 30 (1994) 1-59.

[28] M.G. Hall, Vortex breakdown, Annu. Rev. Fluid Mech. 4 (1) (1972) 195-218.

[29] S. Leibovich, The structure of vortex breakdown, Annu. Rev. Fluid Mech. 10 (1) (1978) 221-246.

[30] T. Sarpkaya, Turbulent vortex breakdown, Phys. Fluids 7 (1995) 2301-2303.

[31] K. Oberleithner, C.O. Paschereit, R. Seele, I. Wygnanski, Formation of turbulent vortex breakdown: Intermittency, criticality, and global instability, AIAA J. 50 (7) (2012) 1437-1452.

[32] S.K. Lele, Compact finite difference schemes with spectral-like resolution, J. Comput. Phys. 103 (1) (1992) 16-42.

[33] J. Berland, C. Bogey, C. Bailly, Low-dissipation and low-dispersion fourth-order Runge-Kutta algorithm, Comput. \& Fluids 35 (2006) 1459-1463.

[34] F. Keiderling, L. Kleiser, C. Bogey, Numerical study of eigenmode forcing effects on jet flow development and noise generation mechanisms, Phys. Fluids 21 (4) (2009) 045106.

[35] T. Poinsot, S.K. Lele, Boundary conditions for direct simulations of compressible viscous flows, J. Comput. Phys. 101 (1992) 104-129.

[36] D.J. Bodony, Analysis of sponge zones for computational fluid mechanics, J. Comput. Phys. 212 (2006) 681-702.

[37] T. Luginsland, How the nozzle geometry impacts vortex breakdown in compressible swirling-jet flows, AIAA J. 53 (10) (2015) 2936-2950.

[38] S. Leibovich, K. Stewartson, A sufficient condition for the instability of columnar vortices, J. Fluid Mech. 126 (1983) 335-356.

[39] J.Z. Wu, A. Xiong, Y. Yang, Axial stretching and vortex definition, Phys. Fluids 17 (2005) 038108.

[40] M.A. Herrada, M. Perez-Saborid, A. Barrero, Nonparallel local spatial stability analysis of pipe entrance swirling flows, Phys. Fluids 16 (2004) 2147-2153.

[41] R. Fernandez-Feria, C. Del Pino, The onset of absolute instability of rotating Hagen-Poiseuille flow: A spatial stability analysis, Phys. Fluids 14 (2002) 3087-3097.

[42] S. Imao, M. Itoh, Y. Yamada, Q. Zhang, The characteristics of spiral waves in an axially rotating pipe, Exp. Fluids 12 (4) (1992) 277-285.

[43] W.O. Criminale, T.L. Jackson, R.D. Joslin, Theory and Computation of Hydrodynamic Stability, in: Cambridge Monographs on Mechanics, Cambridge University Press, 2003.

[44] H.A. Vaidya, Ö Ertunç, B.Z. Genç, F. Beyer, Ç Köksoy, A. Delgado, Numerical simulations of swirling pipe flows- decay of swirl and occurrence of vortex structures, J. Phys.: Conf. Ser. 318 (6) (2011) 062022.

[45] O. Kitoh, Experimental study of turbulent swirling flow in a straight pipe, J. Fluid Mech. 225 (1991) 445-479.

[46] S.K. Lele, Compressibility effects on turbulence, Annu. Rev. Fluid Mech. 26 (1) (1994) 211-254.

[47] J.H. Faler, S. Leibovich, An experimental map of the internal structure of a vortex breakdown, J. Fluid Mech. 86 (1978) 313-335.

[48] L. Facciolo, P.H. Alfredsson, The counter-rotating core of a swirling turbulent jet issued from a rotating pipe flow, Phys. Fluids 16 (2004) L71-L73.

[49] L.A. Dávalos-Orozco, G. Ruiz-Chavarría, Hydrodynamic instability of a fluid layer flowing down a rotating cylinder, Phys. Fluids A 5 (10)(1993) 2390.

[50] S.B. Müller, L. Kleiser, Viscous and inviscid spatial stability analysis of compressible swirling mixing layers, Phys. Fluids 20 (1) (2008) 4103.

[51] G.L. Brown, J.M. Lopez, Axisymmetric vortex breakdown. II-Physical mechanisms, J. Fluid Mech. 221 (1990) 553-576.

[52] L. Lesshafft, P. Huerre, P. Sagaut, Frequency selection in globally unstable round jets, Phys. Fluids 19 (2007) 054108.

[53] B. Hof, C.W.H. van Doorne, J. Westerweel, F.T.M. Nieuwstadt, H. Faisst, B. Eckhardt, H. Wedin, R.R. Kerswell, F. Waleffe, Experimental observation of nonlinear traveling waves in turbulent pipe flow, Science 305 (5690) (2004) 1594-1597. 